\title{
Neuroimaging with magnetoencephalography: A dynamic view of brain pathophysiology
}

Abbreviated Title: MEG Imaging of Neural Pathophysiology

Tony W. Wilson ${ }^{\mathrm{a}, \mathrm{b}, \mathrm{c}, \mathrm{CA}}$, Elizabeth Heinrichs-Graham ${ }^{\mathrm{a}, \mathrm{b}}$, Amy L. Proskovec ${ }^{\mathrm{b}, \mathrm{d}}$, \& Timothy J. McDermott $^{\mathrm{a}, \mathrm{b}}$

a Department of Pharmacology \& Experimental Neuroscience, University of Nebraska Medical Center (UNMC), Omaha, NE, USA

${ }^{\mathrm{b}}$ Center for Magnetoencephalography, UNMC, Omaha, NE, USA

${ }^{\mathrm{C}}$ Department of Neurological Sciences, UNMC, Omaha, NE, USA

${ }^{\mathrm{d}}$ Department of Psychology, University of Nebraska - Omaha, NE, USA

\section{Corresponding Author:}

Tony W. Wilson, Ph.D.

Center for Magnetoencephalography

University of Nebraska Medical Center

988422 Nebraska Medical Center

Omaha, NE 68198

Phone: (402) 552-6431

Fax: (402) 559-5747

Email: tony.w.wilson@gmail.com

Keywords: MEG, oscillation, ADHD, autism, HAND, HIV, PTSD, Parkinson's, cerebral palsy, aging, motor, gamma, beta, alpha, connectivity 


\begin{abstract}
Magnetoencephalography (MEG) is a noninvasive, silent, and totally-passive neurophysiological imaging method with excellent temporal resolution ( 1 ms) and good spatial precision ( 3-5 mm). In a typical experiment, MEG data are acquired while healthy controls and/or patients with neurologic or psychiatric disorders perform a specific cognitive task and/or receive sensory stimulation. The resulting data are generally analyzed using standard electrophysiological methods, coupled with advanced image reconstruction algorithms. To date, the total number of MEG instruments and associated users is significantly smaller than comparable human neuroimaging techniques, although this is likely to change in the near future with advances in the technology. Despite this small base, MEG research has made a significant impact on several areas of translational neuroscience, largely through its unique capacity to quantify the oscillatory dynamics of activated brain circuits in humans. This review focuses on the clinical areas where MEG imaging has arguably had the greatest impact, in regard to the identification of aberrant neural dynamics at the regional and/or network-level, monitoring of disease progression, determining how efficacious pharmacological and behavioral interventions modulate neural systems, and the development of neural markers of disease. Specifically, this review covers recent advances in understanding the abnormal neural oscillatory dynamics that underlie Parkinson’s disease, autism spectrum disorders, HIV-associated neurocognitive disorders, cerebral palsy, attention-deficit/hyperactivity disorder, cognitive aging, and posttraumatic stress disorder. MEG imaging has had a major impact on how clinical neuroscientists understand the brain basis of these disorders, and its translational influence is rapidly expanding with new discoveries and applications emerging continuously.
\end{abstract}




\section{Introduction}

Magnetoencephalography (MEG) is a neurophysiological recording method that is primarily utilized in human studies of systems-level brain function. Although the first MEG measurements occurred more than 40 years ago, ${ }^{1}$ the instrumentation and analytical sophistication of the field have dramatically increased over the past decade..$^{2-5}$ At present, MEG is the only noninvasive high-resolution imaging technique that does not rely on vascular responses (i.e., it measures the neurophysiology directly) and the only functional brain imaging method to offer both high spatial (2-5 mm) and temporal resolution $(<1 \mathrm{~ms}$; see Figure 1$) .{ }^{5}$ Another unique advantage of MEG over other noninvasive methods is that it does not require a reference electrode, which greatly simplifies the interpretation and facilitates network modeling and connectivity analyses.

The physical signal quantified in MEG is comprised of the summated magnetic fields produced by ionic currents within active neural regions. Just as electric current passing through power lines produces magnetic fields, ionic currents within brain tissue also produces magnetic fields according to the same physical principles (e.g., "the right-hand rule” of magnetism). ${ }^{6}$ However, these neuromagnetic fields are ultra-minute $\left(10^{-15} \mathrm{~T}\right)$ relative to other magnetic fields that are typically encountered (e.g., the earth's static field is about $10^{-5} \mathrm{~T}$ ), thus MEG recordings are normally carried out in magnetically-shielded rooms using special sensors and/or noisecancellation software. ${ }^{2-6}$ As per the physiological basis of these ionic currents, MEG is primarily sensitive to dendritic currents in populations of pyramidal neurons in the neocortex. ${ }^{6-7}$ However, other cortical neurons also contribute to the signal, as do neural cells in subcortical and cerebellar regions to a lesser extent. ${ }^{7}$ The orientation, depth (i.e., distance from the sensors), and inherent synchronicity of the neural population also affects the strength of MEG signals, although modern high-density MEG systems are generally sensitive to neural responses across the brain. ${ }^{7-8}$ 
Importantly, in MEG, signal measurement occurs at the sensor-level and typical systems are equipped with a large number of sensors (e.g., 300+) arranged in a helmet-like array (see Figure 1). Consequently, it is necessary to transform the sensor/channel measurements into source space (i.e., anatomical coordinates), and this is generally accomplished using head models and optimization algorithms such as minimum-norm estimation, beamforming methods, and/or related source estimation techniques. ${ }^{2-6}$ This process is often termed MEG source reconstruction.

This review focuses on emerging applications of MEG imaging in translational neuroscience. Some of the most exciting and rapidly-growing research areas for MEG will be covered, including the identification and characterization of neural mechanisms underlying autism, attention-deficit/hyperactivity disorder, posttraumatic stress disorder, and HIV-associated neurocognitive disorders. Several MEG groups are also using the technique’s dynamic mapping capabilities to examine working memory processing in aging, aberrant brain circuits in Parkinson's disease, and the dynamics of motor control in healthy participants and those with cerebral palsy. We will review these areas and the most rapidly evolving application, which is likely the use of MEG to characterize therapeutic effects of experimental and FDA-approved pharmacotherapies and behavioral interventions. To limit redundancy, we will review these "pharmaco-MEG" studies in the section focusing on the target disease (e.g., the effect of stimulants in ADHD). Of note, this review is not limited to studies that were published within a specific time range, although the number of MEG studies per year has increased substantially over the past 15 years and this trend is reflected in this review and Figure 2. In addition, we will not cover the accepted clinical applications of MEG, which in the United States currently includes functional mapping of eloquent cortices (e.g., language-related regions) in neurosurgical candidates, and the localization of epileptic foci in patients with intractable/refractory epilepsy. 
There are many good review papers on the role of MEG in epilepsy and functional mapping prior to tumor resection, and we encourage the interested reader to peruse those publications. ${ }^{9-13}$

Before beginning, a few words about the interpretation and nature of MEG results are probably in order. First, with the exception of resting-state recordings, MEG experiments almost always use event-related designs and are laid out very similar to invasive electrophysiology studies in monkeys and rats. Basically, there is a baseline period with no stimulation, followed by the presentation of a stimulus at time 0.0 seconds (s), which leads to an "active" period where the brain's response to the stimulus occurs. This series of events constitutes one trial, and in the typical MEG experiment there are 80-100 trials per condition, per participant. ${ }^{2}$ The MEG signals are recorded continuously throughout the experiment and these trials are aggregated (offline) in each participant to increase the signal-to-noise ratio of the brain response. In interpreting the signals, it is the relative response (active compared with baseline) that is of paramount interest, because neural cells spontaneously fire and cortical regions are constantly undergoing electrical fluctuations that are seemingly unrelated to ongoing processing. Second, over the past decade, there has been an increasing focus on population-level neural oscillatory activity. ${ }^{14}$ In oscillatory analyses, neural activity in discrete frequency bands, including delta (1-4 Hz), theta (4-7 Hz), alpha (8-14 Hz), beta (14-26 Hz), gamma $(30-50 \mathrm{~Hz})$, and high gamma $(>50 \mathrm{~Hz})$ are examined separately to quantify rhythmic changes in the underlying neural population during cognitive processing. ${ }^{14}$ Note that most MEG studies focus on one or two specific frequency bands, based on their hypotheses, and that the precise definition of a band (e.g., alpha) is often determined statistically for a given study; for example, the definition of alpha may vary from 8-12 $\mathrm{Hz}$ to 8$14 \mathrm{~Hz}$ in different studies. Examining such frequency bands separately enhances the signal-tonoise ratio of each, and enables identification of high-frequency neural activity that is often of 
lower amplitude. These high-frequency responses (i.e., gamma) are widely thought to be critical for interregional communication across active brain networks, ${ }^{15}$ and thus characterizing them is of major importance for understanding overall brain function. For clarity, oscillatory analyses rely on largely the same experimental design, except that trials are often longer, with the relative response again being of primary importance (i.e., active period compared with pre-stimulus baseline). As will become apparent, the application of oscillatory analysis methods to MEG signals and subsequent source reconstruction is a powerful method for identifying the dynamics of brain function (see Figure 3), ${ }^{16}$ and has allowed major discoveries in several distinct areas of clinical cognitive neuroscience.

Finally, a brief overview of MEG terminology and metrics is probably necessary to help facilitate understanding of the key aspects of studies discussed below, as some terms in the field are rather counterintuitive. Critically, the most common metric is amplitude or power of the neural signal, and this can expressed for a particular voxel (i.e., cube of tissue) in the brain, a whole brain region, or at the sensor-level. Pseudo-t is another common MEG unit; in these maps, each pseudo-t value reflects the noise-normalized differential power between an active period and a baseline period for a specific voxel. Other common units include the phase of the signal, based on a sensor-level or voxel time series, as well as an array of functional connectivity measures that are computed using the voxel time series data (typically). Such connectivity measures are thought to reflect inter-regional interactions, and common metrics include coherence, phase-locking factor, phase-lag index, and many other indices. Lastly, for oscillatory analyses, two other common terms are event-related desynchronization (ERD) and event-related synchronization (ERS). An ERD refers to a decrease in band-limited power or amplitude relative to a baseline, whereas as an ERS refers to an increase in band-limited power or amplitude. ERD 
and ERS responses are typically expressed in percentage units, reflecting the percent increase or decrease relative to the baseline, and can be computed at the sensor or voxel level. Autism Spectrum Disorders (ASD)

Autism spectrum disorders (ASD) is one of the most active areas of MEG psychiatric and neurological research, and findings to date have provided significant insight into the underlying pathophysiology. Given the volume of literature, we will focus on studies of auditory processing and the resting-state, as there are several recent reviews that focus more broadly on the electrophysiology of autism. ${ }^{17-19}$

Many MEG studies have shown that participants with ASD have significant auditory processing abnormalities. These studies can be largely divided into two separate categories, consisting of timing deficits in the main auditory response and high-frequency gamma (30-50 $\mathrm{Hz}$ ) aberrations. Starting with the gamma findings, Wilson et al. were first to report changes in the gamma-band in response to auditory stimuli in autism. ${ }^{20}$ They observed reduced steady-state gamma responses in children and adolescents with ASD compared with typically-developing controls, and this effect was more pronounced in the left auditory cortices. ${ }^{20}$ Reduced auditory gamma has also been shown in adults with ASD, and reduced phase-locking in the gamma range has been reported for both children and adults with ASD in MEG studies. ${ }^{21-23}$ Interestingly, both the early auditory gamma response and the steady-state response are impaired in unaffected firstdegree relatives of persons with ASD, suggesting that these findings might be useful as endophenotypes. ${ }^{21,24}$ While the findings of gamma abnormalities have emerged more recently, latency differences in auditory processing have been appreciated for some time. In the standard paradigm, auditory tones (e.g., $1 \mathrm{kHz}$ ) are presented using an oddball paradigm to encourage attention to the stimuli, and in the analysis the focus is on the $100 \mathrm{~ms}$ latency response (the 
M100). Multiple MEG studies have shown delayed peak latencies in participants with ASD compared with age matched controls, ${ }^{22,25-26}$ and a recent study connected this delayed latency in ASD to gamma abnormalities during the pre-stimulus baseline. ${ }^{23}$ Thus, these two categories of deficits may be on the eve of convergence. Finally, one recent study showed that an effective behavioral intervention for adolescents with ASD modulates gamma abnormalities, which supports a direct connection between these neural responses and the observed symptomatology. ${ }^{27}$ In regard to resting-state activity, an early MEG study of children with ASD showed elevated delta, theta, alpha, and high-frequency activity compared with typically-developing children in many regions of the brain, but that only alpha frequency responses in the parietal and anterior temporal regions correlated with symptom severity. ${ }^{28}$ More recent studies have suggested that fewer regions are involved in resting-state ASD aberrations, and that interregional connectivity differences may be critical. ${ }^{29-32}$ For example, Edgar and colleagues investigated a large group of ASD and typically-developing children and found that alpha activity was stronger in the sensorimotor and parietal association cortices of the ASD group, and that alpha power in these regions correlated with scores on a social responsiveness scale. ${ }^{30}$ Group differences were not observed in other areas, although the well-known correlation between peak occipital alpha frequency and age was notably missing in children with ASD. ${ }^{30}$ Connectivity studies using MEG have found hyper-connectivity among frontal, temporal, and subcortical regions in the beta and gamma frequencies of children with ASD, including an orbitofrontal, subcortical, and temporal network implicated in social cognition. ${ }^{31}$ The same study also found that parietal and occipital regions were hypo-connected to most brain regions in the theta and alpha ranges in the ASD compared to typically-developing group, and that children with ASD had altered network topology at both global and local levels. ${ }^{31}$ Finally, another recent MEG study showed that the 
direction of abnormality (i.e., hyper- or hypo-connectivity) in ASD was strongly dependent on the precise frequency band and whether the network included a region in the frontal lobes, with some networks showing opposite effects (hyper/hypo) depending on the precise frequency range being examined. ${ }^{32}$ The findings of Kitzbichler and colleagues ${ }^{32}$ were of major importance, as they provided critical insight to the seemingly contradictory findings of hyper- and hypoconnectivity in ASD, which extend beyond the MEG literature and involve numerous functional MRI (fMRI) and electroencephalography (EEG) studies.

Despite these breakthroughs, the neuronal mechanisms that underlie ASD are not fully understood, as much remains to be discovered. One critical question is how the well-established deficits in auditory processing are related to the resting-state findings and the symptomatology of ASD. While sensory processing abnormalities are an accepted component of ASD, the overall symptom profile is much broader and includes a key social component. Thus, identifying the basic links amongst these neural alterations and symptoms will be critical for future MEG studies and help move the field towards defining a MEG-based neural marker of ASD.

\section{Attention-Deficit/Hyperactivity Disorder (ADHD)}

Attention-deficit/hyperactivity disorder (ADHD) is the most common neurobehavioral disorder in children and adolescents and, for about $65 \%$ of affected children, at least some impairing symptoms will persist into adulthood. ${ }^{33-34}$ While there are only a handful of MEG studies that have investigated ADHD, almost all have taken a pharmaco-MEG approach and evaluated the same patients before and after stimulant-based medications, which are by far the most common treatment for children and adults with ADHD. ${ }^{34-35}$ MEG is uniquely suited for such investigations, as caffeine and other stimulants are robust vasoconstrictors that are known to modulate the fMRI signal through vascular effects alone (i.e., caffeine can alter the fMRI signal 
through vascular changes that are independent of neuronal function), ${ }^{36-39}$ whereas MEG measures the neural signal directly and thus is immune to vascular biases. ${ }^{2-4}$

One early MEG study of ADHD focused on somatosensory processing and found that adults with ADHD had reduced alpha and beta oscillatory responses in the somatosensory cortices following electrical stimulation of the median nerve relative to matched controls. ${ }^{40}$ Such findings suggest that the basic sensory interface is altered in ADHD, and this group proposed that such deficits likely influence higher-order processing. ${ }^{40}$ Another study used a classic oddball paradigm and found evidence of impaired engagement of the ventral visual pathway in adults with ADHD. ${ }^{41}$ They argued that the stimulus-driven reorienting system was defective in ADHD. Several other ADHD studies have focused uniquely on high-frequency gamma activity due to its proposed role in network-level communication and connectivity. ${ }^{15,42-45}$ One used a long duration (i.e., minutes) time estimation task and found that un-medicated adults with ADHD were impaired at judging time, and had significantly reduced gamma activity relative to healthy controls across a network of brain regions that included prefrontal cortices. ${ }^{46}$ Participants in this study were also scanned in the medicated state, and the results indicated significantly improved performance in the time estimation task, but persistent deficits in gamma activity in most brain regions. ${ }^{46}$ A related study of sensory processing investigated gamma activity in the primary auditory cortices, and reported reduced auditory gamma in the un-medicated state, along with significantly increased gamma following medication. ${ }^{47}$ Interestingly, medicated patients did not statistically differ from controls in this study, which may suggest that stimulant medications act to normalize connectivity via modulating local gamma activity. Related findings have emerged from MEG studies of attention in patients with ADHD. For example, one study of auditory attention found that stimulants modulate gamma activity in frontal and parietal areas ${ }^{48}$ whereas 
another found hyper-connectivity between prefrontal and auditory cortices in un-medicated adults with ADHD, which was significantly decreased following stimulant administration. ${ }^{49}$ One other study focused on alpha differences during visual attention and found that decreased alpha lateralization correlated with impaired performance in patients with ADHD. ${ }^{50}$

Finally, there have also been several MEG studies of resting-state activity in ADHD. One focused on the default-mode network and found that un-medicated adults with ADHD had reduced neural activity compared to demographically-matched controls in the medial prefrontal cortices, but not other nodes in the network. ${ }^{51}$ This study also found that administering stimulant medications significantly increased 8-14 Hz alpha activity in the medial prefrontal area, and decreased inattention symptoms. ${ }^{51} \mathrm{~A}$ later pharmaco-MEG study of the default-mode network found an array of connectivity differences between un-medicated patients with ADHD and controls that varied by frequency, including hypo-connectivity between medial prefrontal and posterior cingulate cortices, and hyper-connectivity between posterior regions of the default mode network (see Figure 4). ${ }^{52}$ This study also found that, following stimulant administration, all connectivity differences in the default mode network dissipated and there were no significant connectivity differences between the two groups. ${ }^{52}$ This finding was particularly important as it suggests that ADHD medications may normalize connectivity in active brain networks, and thus reconciles data from other studies that focused on the local amplitude of neural activity and found decreased symptoms but residual brain abnormalities following medication delivery. While future studies are certainly needed, the current data supports abnormal functional connectivity in ADHD and that stimulant medications may normalize connectivity and suppress symptoms by modulating local high-frequency gamma activity. 
Although the collection of MEG studies in this area have provided critical insight, one major area a ADHD symptomatology has yet to be systematically examined. Essentially, response variability is a hallmark feature of ADHD and the unique characteristics of MEG imaging make it an ideal modality for identifying the neural mechanisms. One particularly attractive hypothesis is that response variability is related to current gamma levels in active cortical regions, and that the abnormally reduced gamma in ADHD cannot reliably sustain interregional communication during active cognitive processing. This hypothesis could be tested with MEG and future studies should consider this, and alternative mechanisms, in investigations that identify the precise neural dynamics that underlie response variability in ADHD. Post-Traumatic Stress Disorder (PTSD) Posttraumatic stress disorder (PTSD) is a complex diagnosis involving re-experiencing, avoidance, negative mood and cognition, and arousal symptoms. ${ }^{53}$ The lifetime prevalence of PTSD in the United States is roughly $7-9 \%,{ }^{54-55}$ but prevalence rates among recent combat veterans returning from wars in Iraq and Afghanistan has been estimated at $13 \%$ to $22 \%{ }^{56-57}$ Greater combat exposure has been generally associated with greater PTSD symptomatology. ${ }^{56-57}$ While MEG studies of PTSD are historically rare, there has been a significant wave of studies over the past few years as interest and funding in this area has sharply increased. Probably the most common findings involve an advanced analysis method that looks at the overall pattern of synchronous neural interactions, with the long-term goal of identifying a MEG biomarker for PTSD ${ }^{58-62}$ These studies have shown that the right parieto-occipital and temporal cortices miscommunicate with other brain regions in PTSD. ${ }^{58-62}$ Moreover, a recent study using this method in over 200 participants showed that global neural interactions were significantly down-modulated with increasing lifetime trauma scores in resilient veterans, but not those with 
PTSD, and that this effect was strongest in small networks in the right superior temporal gyrus. ${ }^{62}$ Studies using more conventional methods have also found aberrant right hemispheric activity in PTSD, including increased slow wave activity in right frontal cortices during rest ${ }^{63}$ and increased right prefrontal activity during the processing of aversive relative to positive and neutral images. ${ }^{64}$ Enhanced processing of trauma stimuli (i.e., combat-related words) in veterans with PTSD compared with non-PTSD veterans was reported in a later study, although the main neural difference was in visual cortices. ${ }^{65}$ However, a recent study demonstrated aberrant processing of non-threatening touch (i.e., an air-puff delivered to a finger) in veterans with PTSD compared to those without, with significant differences in primary somatosensory areas and the right prefrontal cortices. ${ }^{66}$ This finding suggests aberrant sensory responses may be a more general phenomenon that is not tied directly to the valence of the stimuli, and again implicates right hemispheric cortices. Of note, this group also found that the magnitude of neural activity in prefrontal and somatosensory cortices was correlated with symptom severity, indicating that those with the most abnormal response had the most severe symptoms. ${ }^{66}$ A new study of verbal working memory from our laboratory reached a similar conclusion, as veterans with PTSD exhibited normal responses during both encoding and maintenance operations in left hemispheric language regions, but disturbed responses in right inferior frontal, right supramarginal and superior temporal cortices throughout the majority of encoding and maintenance periods (see Figure 5) ${ }^{67}$ As with other studies, these right hemispheric responses correlated with PTSD symptom severity, and we propose that such activity in homologue areas reflects compensatory processing in veterans with PTSD. ${ }^{67}$ In a follow-up study, we showed that attention training treatment sharply reduced PTSD symptom severity and largely normalized neural activity in these same brain regions during verbal working memory processing. ${ }^{68}$ 
Finally, several studies have examined functional connectivity in veterans with PTSD, and these studies have found abnormal long range hyper-connectivity in the high gamma band of patients with PTSD during rest, ${ }^{69}$ as well as theta hyper-connectivity in the right parietal cortices of veterans with PTSD during a mental flexibility task. ${ }^{70}$ In both cases, the degree of aberrant connectivity was correlated with cognitive outcome variables and symptom scales. ${ }^{69-70}$ Interestingly, a recent study from the same group showed that veterans with PTSD have increased inter-network synchronization in the gamma and high gamma range relative to those without PTSD, and that symptom severity was correlated with gamma activity in the salience network. $^{71}$

PTSD is definitely one of the fastest growing research areas for MEG, and this is likely to continue as the method is ideal for studies of brain dynamics. MEG studies to date have identified altered dynamics across widespread cortical regions and networks in patients with PTSD (mostly veterans), although findings of right hemispheric abnormalities involving frontal, prefrontal, and parietal cortices are by far the most common. Some of these findings appear to be very consistent and may hold promise as MEG-based biomarkers, which would be a significant breakthrough as distinguishing PTSD from other disorders is not trivial. Studies that connect MEG markers and clinical symptom scales are particularly needed as the field moves in this direction.

HIV-Associated Neurocognitive Disorders (HAND)

The advent of combination antiretroviral therapy shifted the nature of HIV-infection from a terminal illness to a chronic manageable condition, with a life expectancy that has gradually approached that of seronegative persons. ${ }^{72-73}$ However, HIV-infected patients remain at a significantly elevated risk of developing HIV-associated neurocognitive disorders (HAND), with 
$35-70 \%$ of all patients exhibiting at least subtle impairments on tests of neuropsychological function. ${ }^{74-81}$ Neuropsychological studies of HAND have documented aberrations across a broad range of functional domains (e.g., attention, fine motor), but the basic pathophysiology remains unresolved. Furthermore, despite its high prevalence, there are no diagnostic tests or biomarkers that can precisely detect HAND, and diagnoses are currently made by exclusion of other factors.

MEG studies in this area have tended to focus on identifying the effects of HIV-infection on brain function and the neural processes that underlie HAND. While only a few MEG studies have been conducted to date, their findings have provided significant insight. For example, an MEG study of motor control demonstrated that HIV-infected patients had reduced beta responses $(14-28 \mathrm{~Hz})$ prior to movement onset in the supplementary motor area and bilateral primary motor cortices (stronger on the side contralateral to movement) relative to demographically-matched controls.$^{82}$ HIV-infected patients also had increased activity in bilateral prefrontal cortices, which likely reflected compensatory processes serving movement planning, as significant correlations were observed between the amplitude of such activity and performance on the Trail Making Test $(\mathrm{A}+\mathrm{B}) \cdot{ }^{82}$ An MEG study of visual attention also found abnormal activity in the right prefrontal cortices, as well as the frontal eye-fields of HIV-infected older adults compared with matched uninfected controls. ${ }^{83}$ Consistent with the motor control study, the magnitude of neural responses in these areas correlated with neuropsychological performance in the HIV-infected patients. ${ }^{83}$ In regard to resting-state activity, MEG studies have shown deficits in functional connectivity in HIV-infected patients, especially involving right temporo-parietal and occipital cortices, ${ }^{84}$ and reduced resting beta activity in superior parietal regions, posterior cingulate, supplementary motor cortices, and the paracentral lobule. ${ }^{85}$ Finally, a recent multimodal study from our laboratory examined local gray matter volume and tactile processing in older HIV-infected and 
uninfected participants. We found reduced neural responses in the left postcentral gyrus (contralateral to stimulation) shortly after onset of the tactile stimulus in HIV-infected patients, along with increased alpha activity in the prefrontal cortices. ${ }^{86}$ HIV-infected patients also had reduced gray matter in parahippocampal, lingual, and other areas, as well as a region of the left postcentral gyrus that spatially coincided with the abnormal MEG response (see Figure 6). ${ }^{86}$ This latter finding suggests that some of the functional MEG aberrations observed in HIV disease may reflect alterations in the underlying neural structure, whereas others are more purely functional in nature (e.g., prefrontal cortices). Of particular clinical interest, we computed sensitivity and specificity indices for HAND using the peak amplitude values of the two main MEG findings (i.e., left postcental gyrus and prefrontal cortices) in conjunction, and found that the sensitivity and specificity for HAND were over $85 \%{ }^{86}$ These findings strongly suggest that MEG metrics may serve as powerful classifiers in the context of HAND and HIV-associated brain dysfunction. Furthermore, another recent MEG study reported excellent test-retest reliability after $\sim 24$ weeks in both HIV-infected and uninfected participants, ${ }^{87}$ which of course is a critical property for any biomarker of disease.

In summary, MEG studies of the neural alterations associated with HIV-infection are rare, but have increased substantially over the past three years. These studies have repeatedly implicated the prefrontal cortices, right more often than left, as a key dysfunctional hub across several different tasks. These data and those reflecting altered sensory processing in HIVinfection provide clear support for the utility of MEG in identifying and characterizing the pathophysiology of interest, and the technique's potential as a future test for HAND. Future studies should examine the impact of disease duration (time since diagnosis), substance abuse, and aging, as these factors may be particularly important in understanding the pathophysiology. 


\section{Parkinson's Disease (PD)}

Parkinson's disease (PD) is the second most common neurodegenerative disorder in the world, it is progressive and has an overall prevalence of $1-2 \%$ in the United States. ${ }^{88-90}$ There is a wealth of literature that describes the motor abnormalities commonly observed in patients with PD, ${ }^{91-95}$ which include muscle rigidity, instability, hypo- or bradykinesia (i.e., lack of, or slowness of movement), and resting tremor. While the exact mechanisms are unknown, these symptoms likely arise from the progressive degeneration of dopaminergic neurons in the substantia nigra pars compacta $(\mathrm{SNpc}){ }^{96}$

Some of the most important MEG studies of patients with PD have involved recording MEG simultaneously with local-field potentials (LFP) from within the subthalamic nucleus (STN) during deep-brain stimulation (DBS) surgery in patients with severe PD. ${ }^{97-102}$ These simultaneous MEG-LFP recordings have allowed conclusions about how the subcortical pathophysiology transcends and affects the cortex. One of the most common findings from this work has been pathological synchrony between neurons within the STN, as well as between STN and cortical motor neurons, both of which are normalized by DBS and/or dopamine replacement therapy. This hyper-synchrony is frequency specific, with tremor-related alterations being found predominantly in the theta $(4-7 \mathrm{~Hz})$ and alpha bands $(8-12 \mathrm{~Hz})$, corresponding to the frequency of Parkinsonian tremor and its harmonic, while more general motor deficits are related to beta (14-30 Hz) and gamma (> $30 \mathrm{~Hz}$ ) synchrony. For example, Hirschmann and colleagues found that during isometric contraction, coherence between the STN and the primary motor cortex was dominant in the beta rhythm, and that this synchrony was significantly diminished and motor symptoms improved following administration of dopaminergic medication. ${ }^{102}$ These studies, coupled with knowledge from purely invasive recordings (for a review, see ${ }^{103}$ ), support a model 
of subcortico-cortical oscillatory deviations in $\mathrm{PD}$, where neural oscillations become hypersynchronous in the STN and eventually impair motor function by engulfing other structures within the motor network into a state of excessive hyper-synchronization. ${ }^{104}$

While important in their own right, studies that have employed simultaneous LFP-MEG methodology have at least two major limitations. First, due to the invasive nature of LFP recordings, comparisons involving a healthy control sample are simply not possible. Secondly, patients who are eligible for DBS surgery generally exhibit very severe symptoms and thereby are representative of only a small percentage of the overall PD population. Thus, MEG studies of patients with mild-to-moderate PD provide a nice complement, and offer broader implications as to the role of oscillations in PD. ${ }^{105-115}$ One such study found increased beta connectivity between the primary motor cortices and supplementary motor area during isometric contraction, which correlated with measures of symptom severity and was reduced following administration of dopaminergic medication. ${ }^{106}$ Another study from the same group found widespread resting-state synchrony between the primary motor cortices, premotor region, supplementary motor area, and parietal regions in theta and alpha frequencies that was directly related to tremor amplitude in patients with PD. ${ }^{107}$ Recent work from our laboratory confirmed that un-medicated patients with PD have pathologically high synchrony between the primary motor cortices, and further showed that these patients have significantly diminished beta amplitude relative to demographicallymatched controls during the resting-state. ${ }^{112}$ Interestingly, administration of dopaminergic medication selectively ameliorated these differences in beta amplitude. ${ }^{112}$

Work from our laboratory was also the first to utilize the temporal precision of MEG to systematically investigate the effects of PD on motor physiology before, during, and after transient movement. ${ }^{113}$ Essentially, prior to movement onset, there is a desynchronization in the 
beta band, termed the peri-movement beta ERD, which starts approximately $0.5 \mathrm{~s}$ before motor onset and continues throughout movement execution. Following movement termination, there is a strong resynchronization in the beta band known as the post-movement beta rebound (PMBR) response. ${ }^{113-121}$ The PMBR reaches its maximum amplitude 0.5 to $1.0 \mathrm{~s}$ after the termination of movement, and continues for about $1.0 \mathrm{~s}$ before returning to baseline levels. These responses are at least partially spatially distinct; the peri-movement beta ERD generally involves the bilateral primary sensorimotor cortices, supplementary motor area, parietal lobe and the cerebellum, whereas the PMBR has been reported in the sensorimotor cortices, supplementary motor area, premotor, and medial prefrontal regions. ${ }^{113-121}$ The temporal and spatial precision of MEG is optimal for studying this pattern of responses in health and in patients with movement disorders such as PD. By exploiting these optimal properties, we showed that the peri-movement beta ERD response in the primary motor cortices was selectively aberrant in patients with PD compared to controls (see Figure 7). ${ }^{113}$ Since then, other labs have identified and selectively modulated the peri-movement beta ERD response in PD. ${ }^{122-123}$ Of note, one of these studies demonstrated differences in the millisecond time course of the peri-movement beta ERD, and suggested that patients with PD exhibit neural dynamics reflective of a reactive, rather than proactive, movement planning process. ${ }^{122}$ This and other supporting evidence suggests that this motorrelated oscillatory response may serve as a biomarker of symptom severity and disease progression.

While future studies are certainly needed, MEG studies in PD have provided critical insight into how this disease affects brain physiology, especially within the subcortical-cortical motor network. One major question in the field is how specific these neural abnormalities are to PD, as the symptom profile of PD overlaps with several different disorders (e.g., progressive 
supranuclear palsy). MEG studies showing that the neurophysiological aberrations are unique to PD will be exceptionally important, and establish a foundation for developing disease specific markers that could potentially be used to identify patients for future clinical trials.

Cerebral Palsy (CP)

Cerebral palsy (CP) is one of the most prevalent pediatric neurologic conditions diagnosed in the United States, ${ }^{124}$ with almost four out of every 1000 children being affected. It results from a defect or insult to the immature developing brain and, ${ }^{125}$ although the neurologic damage does not progressively worsen, it often results in neuromuscular impairments that limit the child's mobility. The total lifetime economic burden to our society is estimated to be about one million dollars per child, with a major portion of these funds directed at interventions that are aimed at improving the child's mobility. ${ }^{126}$ MEG studies in this area have primarily focused on identifying the contribution of central mechanisms (i.e., the brain) to the observed motor deficits in children and adolescents with CP.

Early MEG studies in this area focused on the primary somatosensory response evoked by electrical stimulation of the tibial nerve. These studies showed that children with CP had sharply reduced response amplitudes compared to typically-developing controls in the contralateral somatosensory cortices, especially for the earliest component. ${ }^{127}$ A later study showed that body-weight supported treadmill training improved lower extremity motor function, and modulated these same MEG somatosensory responses in children with CP. ${ }^{128}$ More recent work in this area has examined the oscillatory dynamics of somatosensory processing, and these studies have shown that children with CP have abnormal neural synchronization within sensorimotor cortices following tactile stimulation of the foot. ${ }^{129-130}$ Specifically, children with CP exhibited decreased synchronization in the theta-alpha range $(4-14 \mathrm{~Hz})$ in response to tactile 
stimulation, whereas typically-developing controls showed robust increases in theta-alpha synchronization in the same medial sensorimotor cortices. ${ }^{129}$ Interestingly, the strength of neural synchronization in the somatosensory cortices, induced by tactile stimulation, has also been connected to ankle strength, step length, and walking speed in children with CP, which suggests a direct connection between these sensory processing measures and motor performance. ${ }^{130} \mathrm{~A}$ related study of particular interest showed that theta-alpha synchronization in the somatosensory cortices was normal in children with CP following tactile stimulation of the hand. ${ }^{131}$ Since the children in this study did not have upper extremity impairments, these findings may suggest that theta-alpha synchronization is selectively disturbed in CP and is only abnormal in neural regions that serve severely impaired limbs.

Finally, one recent MEG study examined motor control of the knee joint in children and adolescents with CP. ${ }^{132}$ This groundbreaking study precisely quantified movement onset and examined the oscillatory dynamics of motor planning, execution, and termination. Their findings showed that patients with CP had significantly stronger beta ERD responses during the motor planning stage compared to typically-developing controls, along with significantly weaker gamma responses during movement execution. ${ }^{132}$ These investigators proposed that the stronger beta ERD reflected increased planning activity, as patients tried to compensate for their motor impairments, and that the reduced gamma response indicated that this planning was not fully successful, and ultimately cascaded into the execution and termination stages. ${ }^{132}$ These findings may suggest a specific therapeutic target for designing new physical therapy interventions. ${ }^{132-133}$ While this study of motor activity in CP provided critical data, much remains to be discovered and MEG will certainly be involved. Understanding the neural mechanisms underlying motor impairments will be strongly advanced if the temporal dynamics of neuronal circuits can be 
quantified in real time along with the movement parameters. Furthermore, the silent nature of MEG and other unique properties makes it an ideal technique for studying children and adolescents with CP, as these participants are easily startled and often have spasms that make fMRI scanning more tenuous. Future studies will need to identify how the severity of CP affects the neuronal aberrations reported in previous studies, and decipher how age and other factors modulate these findings.

\section{Healthy Aging}

Healthy aging is associated with declines in sensory, perceptual, and cognitive processes. ${ }^{134-135}$ The neurophysiological changes that underlie these declines are not fully understood, and MEG studies in this area have generally focused on identifying and characterizing these changes in the context of sensory stimulation and/or demanding cognitive tasks. Findings from other modalities (e.g., fMRI) suggest that older adults typically utilize greater neural resources relative to younger adults, which may reflect compensatory mechanisms ${ }^{136}$ or neural dedifferentiation. ${ }^{137}$

Perhaps the most commonly studied aging effects in the MEG literature pertain to sensory and perceptual operations, with aging being associated with deficits in such processes. Several MEG studies have examined the neurophysiological underpinnings of healthy aging using auditory processing paradigms, and have shown that older adults generally demonstrate a stronger amplitude response relative to younger adults in superior temporal regions shortly after the onset of an auditory stimulus ( $\sim 50 \mathrm{~ms})$. This accentuated early neural response has been demonstrated in response to complex sounds, ${ }^{138}$ phonetic sounds, ${ }^{139-140}$ spoken words, ${ }^{141}$ pitch, ${ }^{142-143}$ and tones. ${ }^{139,144-145}$ Interestingly, similar age-related differences have been reported in somatosensory MEG studies utilizing median nerve stimulation, with older adults exhibiting an increased early cortical response in the primary somatosensory cortices, ${ }^{146-151}$ especially 
contralateral to the stimulated nerve. ${ }^{146-150}$ Of note, neural responses at later latencies are typically more comparable in strength and do not generally show aging effects in auditory or somatosensory modalities, although there have been exceptions. ${ }^{138,141}$ Together these studies suggest that early hyper-responsivity to sensory stimulation in the elderly may underlie agerelated behavioral deficits in sensory processing. In addition, the latency of these responses and later components is generally delayed in older relative to younger adults, and this may underlie the age-related slowing of sensory and perceptual processes commonly seen.

A handful of MEG resting-state studies have also examined the neural underpinnings of aging. Recent studies have found that global low frequency delta/theta $(0.5-6.5 \mathrm{~Hz})$ activity decreased with increasing age, and that such reductions in bilateral temporal and parietal regions were associated with reduced cognitive performance. ${ }^{152-153}$ MEG studies that have focused on network-level activity have also found reduced delta (2-4 Hz) network size and larger beta/gamma (>16 Hz) network size in older compared with younger adults, with increased beta/gamma connectivity in bilateral temporal lobes being associated with worse performance on visuoconstructive tasks. ${ }^{154-155}$ Taken together, stronger low-frequency oscillations in the restingstate are associated with better executive performance in the elderly, while increased beta/gamma connectivity in the temporal cortices during rest may be deleterious and contribute to neural dedifferentiation in the elderly.

Finally, MEG has been used to investigate age-related differences in the spatiotemporal dynamics underlying higher-order cognitive processes, with working memory perhaps being the most popular. For example, Proskovec and colleagues reported widespread neural activity in left fronto-temporal regions of both young and older adults during the encoding and maintenance periods of a verbal working memory task, but only older adults also engaged the right prefrontal 
cortices during task performance. ${ }^{156}$ Results for the retrieval phase of working memory were reported by Aine et al. ${ }^{157}$ Notably, no age-related differences in working memory performance were observed in either of these studies. ${ }^{156-157}$ Together, these studies support the compensation hypothesis of cognitive aging. ${ }^{136}$ Essentially, older adults recruited more neural resources during working memory performance across encoding, maintenance, and retrieval periods compared with younger adults, while performance was relatively equal between groups. Interestingly, the Proskovec study also found strong alpha $(9-12 \mathrm{~Hz})$ synchronization in the occipital cortices during memory maintenance in both age groups, although this response occurred earlier and encompassed more cortical tissue in older adults (Figure 8). ${ }^{156}$ Occipital alpha synchronization is believed to reflect inhibition of the dorsal visual stream during memory maintenance, which would aid in blocking incoming visual distractions and help preserve memory representations in more anterior cortical regions. ${ }^{158-160}$ Thus, the earlier and more widespread increase in occipital alpha activity in older adults likely reflects an additional compensatory mechanism.

In summary, MEG is an ideal tool for examining the mechanisms of cognitive aging, as its spatiotemporal precision allows age-related delays and slowing in the system to be accurately characterized. While most early investigations focused on sensory processing, MEG studies of higher-order processes are now appearing in the literature and these data will eventually help the field distinguish early signs of dementia from the neuronal signatures of healthy aging. A solid understanding of age-related changes will also enable identification of accelerated cognitive aging, and lead to more controlled neuroimaging investigations of aging subtypes in the future.

\section{Conclusions \& Future Directions}

MEG is an advanced neurophysiological imaging tool with excellent spatiotemporal precision, which makes it an ideal method for studying the brain dynamics that underlie neurologic and 
psychiatric disorders. These unique properties have enabled MEG imaging to make a significant impact on clinical cognitive neuroscience, despite the relative rarity and emergent status of the technique. Specifically, MEG studies have identified aberrations in the auditory system of patients with ASD, including deficient high-frequency gamma activity and deviations in response timing. Several groups are now working to develop an auditory-based MEG biomarker. MEG studies of ADHD have quantified how stimulant medications affect brain function in these patients, and identified connectivity deficits and gamma abnormalities across multiple brain regions. Notably, these studies have suggested that stimulants may normalize connectivity by modulating local gamma activity, which would be consistent with models of how gamma activity affects network-level neural interactions. The role of MEG in understanding PTSD is rapidly expanding, with the early findings indicating that patients (mostly veterans) have altered oscillatory dynamics across multiple cortical regions, with right frontal and parietal areas being among the most commonly implicated. MEG studies of HIV-infection and HAND have also highlighted deficits in the right prefrontal cortices, although the most exciting findings in this area are likely the sensitivity and specificity data, as well as the reliability data, showing that MEG metrics may serve as a diagnostic biomarker. In studies of PD, MEG has already had a huge impact in quantifying beta activity across the motor network, both in a surgical context (DBS) and in more mild cases of PD. Several recent studies have started to dissect how beta oscillations in the motor circuit separately affect motor planning, execution, and termination stages, which will ultimately lead to understanding how beta activity deficits interact with motor symptoms. MEG studies of children with CP are also expanding rapidly, as the silent MEG recording environment is ideal for this population. Critical findings thus far have included beta abnormalities during motor planning, as well as major somatosensory processing deficits. 
Finally, MEG studies of cognitive aging have shown considerable promise, as the excellent temporal resolution of MEG is ideal for discerning age-related changes in neural timing.

Findings so far include early sensory processing deficits, as well as strong compensatory activity in right hemispheric homologue regions during higher-order cognitive tasks. MEG markers of healthy versus pathological aging are also under development, although much work remains to be completed in this arena.

The use of MEG has grown rapidly over the past decade, especially in the past few years, and this is very likely to continue through the next decade, with the increasing focus on brain dynamics in clinical cognitive neuroscience, and dedicated funding opportunities like President Obama's Brain Initiative in the United States and the European Union’s Human Brain Project. This growth will include efforts to develop new MEG-based biomarkers of disease, as well as markers that can be used to monitor disease progression and therapeutic responses to treatment (i.e., pharmaco-MEG). In the future, MEG may see action in the early stages of drug development to ascertain where drugs are primarily acting. This seems especially likely for disorders where certain brain areas are known to underlie key aspects of the symptomatology, such as the frontal and prefrontal cortices in ADHD. Thus, MEG will likely appear in many new venues in the coming years, as it continues to benefit from the scientific community's growing focus on translational research. Lastly, new innovative MEG sensor designs are now emerging, ${ }^{161}$ and these hold promise for significantly improving the spatial resolution of MEG $(<$ $1 \mathrm{~mm}$ ) while lowering instrumentation costs. If successful, the number of MEG sites and the pace of discovery could rapidly expand. 


\section{Acknowledgements}

This work was supported by NIH grant R01 MH103220 (TWW), NSF grant \#1539067 (TWW), the Shoemaker Prize from the University of Nebraska Foundation (TWW), a Kinman-Oldfield Award for Neurodegenerative Research from the University of Nebraska Foundation (TWW), and a grant from the Nebraska Banker's Association (TWW). The Center for Magnetoencephalography at the University of Nebraska Medical Center was founded through an endowment from an anonymous donor. The funders had no role in study design, data collection and analysis, decision to publish, or preparation of the manuscript. Drs. Wilson and HeinrichsGraham, Ms. Proskovec, and Mr. McDermott have read the journal's policy on conflicts of interest and have no potential conflicts of interest to disclose. In addition, all authors have read the journal's authorship agreement and fulfilled all criteria for authorship. 


\section{References}

1. Cohen, D.S. Magnetoencephalography: Evidence of magnetic fields produced by alpha rhythm currents. Science 1968;161:784-786.

2. Wilson, T.W. (2014). Noninvasive neurophysiological imaging with magnetoencephalography. In H. Xiong \& H.E. Gendelman (Eds), Current laboratory methods in neuroscience research (pp.293-311). New York: Springer Press.

3. Supek, S., Aine, C.J. (Eds). (2014). Magnetoencephalography: From signals to dynamic cortical networks. Verlag: Springer Press.

4. Hansen, P.C., Kringelbach, M.L., Salmelin, R. (Eds). (2010). MEG: An introduction to the methods. New York: Oxford University Press.

5. Hari, R., Salmelin, R. Magnetoencephalography: From SQUIDs to neuroscience. Neuroimage 20th anniversary special edition. Neuroimage. 2012;61(2):386-96.

6. Hämäläinen, M., Hari, R., Ilmoniemi, R.J., Knuutila, J., Lounasmaa, O.V. Magnetoencephalography -Theory, instrumentation, and applications to noninvasive studies of the working human brain. Rev Mod Phys. 1993;65(2):413-495.

7. Murakami, S., Okada, Y. Contributions of principal neocortical neurons to magnetoencephalography and electroencephalography signals. J Physiol. 2006;575:925-936.

8. Hillebrand , A., Barnes, G.R. A quantitative assessment of the sensitivity of whole-head MEG to activity in the adult human cortex. Neuroimage. 2002;16:638-650.

9. Bagić, A. Look back to leap forward: The emerging new role of magnetoencephalography (MEG) in nonlesional epilepsy. Clin Neurophysiol. 2015 May15.doi:

10.1016/j.clinph.2015.05.009. [Epub ahead of print] Review.

10. Stufflebeam, S.M. Clinical magnetoencephalography for neurosurgery. Neurosurg Clin N Am. 2011;22(2):153-167, vii-viii.

11. Stufflebeam, S.M., Tanaka, N., Ahlfors, S.P. Clinical applications of magnetoencephalography. Hum Brain Mapp. 2009;30(6):1813-1823.

12. Stefan, H., Rampp, S., Knowlton, R.C. Magnetoencephalography adds to the surgical evaluation process. Epilepsy Behav. 2011;20(2):172-177.

13. Tanaka, N., Stufflebeam, S.M. Clinical application of spatiotemporal distributed source analysis in presurgical evaluation of epilepsy. Front Hum Neurosci. 2014;8:62. Review.

14. Jensen, O., Spaak, E., Zumer, J.M. (2014). Human brain oscillations: From physiological mechanisms to analysis and cognition. In S. Supek \& C.J. Aine (Eds), 
Magnetoencephalography: From signals to dynamic cortical networks (pp. 359-403). Verlag: Springer Press.

15. Uhlhaas, P.J., Pipa, G., Lima, B., Melloni, L., Neuenschwander, S., Nikolić, D., Singer, W. Neural synchrony in cortical networks: history, concept and current status. Front Integr Neurosci. 2009;3:17.

16. Schoffelen, J.M., Gross, J. (2014). Studying dynamic neural interactions with MEG. In S. Supek \& C.J. Aine (Eds), Magnetoencephalography: From signals to dynamic cortical networks (pp. 359-403). Verlag: Springer Press.

17. Rojas, D.C., Wilson, L.B. $\gamma$-band abnormalities as markers of autism spectrum disorders. Biomark Med. 2014;8(3):353-68.

18. McFadden, K.L., Rojas, D.C. (2013). Electrophysiology of autism. In Recent Advances in Autism Spectrum Disorders - Volume II. Michael Fitzgerald (Ed.), ISBN: 978-953-51-1022-4, InTech, DOI: 10.5772/54770.

19. Rojas, D.C. The role of glutamate and its receptors in autism and the use of glutamate receptor antagonists in treatment. J Neural Transm. 2014;121(8):891-905.

20. Wilson, T.W., Rojas, D.C., Reite, M.L., Teale, P.D., Rogers, S.J. Children and adolescents with autism exhibit reduced MEG steady-state gamma responses. Biol Psychiatry 2007;62:192197.

21. Rojas, D., Maharajh, K., Teale, P., Rogers, S. Reduced neural synchronization of gammaband MEG oscillations in first-degree relatives of children with autism. BMC Psychiatry. 2008;8(1):66.

22.Gandal, M.J., Edgar, J.C., Ehrlichman, R.S., Mehta, M., Roberts, T.P.L., Siegel, S.J. Validating $\gamma$ oscillations and delayed auditory responses as translational biomarkers of autism. Biological Psychiatry. 2010;68(12):1100-1106.

23. Edgar, J.C., Khan, S.Y., Blaskey, L., Chow, V.Y., Rey, M., Gaetz, W., Cannon, K.M., Monroe, J.F., Cornew, L., Qasmieh, S., Liu, S., Welsh, J.P., Levy, S.E., Roberts, T.P. Neuromagnetic oscillations predict evoked-response latency delays and core language deficits in autism spectrum disorders. J Autism Dev Disord. 2015;45(2):395-405.

24. Rojas, D.C., Teale, P.D., Maharajh, K., et al. Transient and steady-state auditory gammaband responses in first-degree relatives of people with autism spectrum disorder. Mol Autism. 2011;2(1):11.

25. Gage, N.M., Siegel, B., Roberts, T.P. Cortical auditory system maturational abnormalities in children with autism disorder: an MEG investigation. Brain Res Dev Brain Res. 2003;144:201209. 
26. Roberts, T.P., Khan, S.Y., Rey, M., Monroe, J.F., Cannon, K., Blaskey, L., Woldoff, S., Qasmieh, S., Gandal, M., Schmidt, G.L., Zarnow, D.M., Levy, S.E., Edgar, J.C. MEG detection of delayed auditory evoked responses in autism spectrum disorders: towards an imaging biomarker for autism. Autism Res. 2010;3:8-18.

27. Van Hecke, A.V., Stevens, S., Carson, A.M., et al. Measuring the Plasticity of Social Approach: A Randomized Controlled Trial of the Effects of the PEERS Intervention on EEG Asymmetry in Adolescents with Autism Spectrum Disorders. J Autism Dev Disord. (2013).

28. Cornew, L., Roberts, T.P., Blaskey L, Edgar JC. Resting-state oscillatory activity in autism spectrum disorders. J Autism Dev Disord. 2012;42(9):1884-1894.

29. Berman, J.I., Liu, S., Bloy, L., Blaskey, L., Roberts, T.P., Edgar, J.C. Alpha-to-gamma phase-amplitude coupling methods and application to autism spectrum disorder. Brain Connect. 2015;5(2):80-90.

30. Edgar, J.C., Heiken, K., Chen, Y.H., Herrington, J.D., Chow, V., Liu, S., Bloy, L., Huang, M., Pandey, J., Cannon, K.M., Qasmieh, S., Levy, S.E., Schultz, R.T., Roberts, T.P.. Restingstate alpha in autism spectrum disorder and alpha associations with thalamic volume. $J$ Autism Dev Disord. 2015;45(3):795-804.

31.Ye, A.X., Leung, R.C., Schäfer, C.B., Taylor, M.J., Doesburg, S.M. Atypical resting synchrony in autism spectrum disorder. Hum Brain Mapp. 2014;35(12):6049-6066.

32.Kitzbichler, M.G., Khan, S., Ganesan, S., Vangel, M.G., Herbert, M.R., Hämäläinen, M.S., Kenet, T. Altered development and multifaceted band-specific abnormalities of resting state networks in autism. Biol Psychiatry. 2015;77(9):794-804.

33. Faraone, S.V., Biederman, J., Mick, E. The age-dependent decline of attention deficit hyperactivity disorder: A meta-analysis of follow-up studies. Psychol Med. 2006;36:159-165.

34. Center for Disease Control and Prevention. Mental health in the United States: Prevalence of diagnosis and medication treatment for attention-deficit/hyperactivity disorder - United States, 2003. MMWR 2005;54(34):842-847.

35. Coghill, D., Seth, S. Osmotic, controlled-release methylphenidate for the treatment of ADHD. Expert Opin Pharmacother 2006;7:2119-2138.

36. Laurienti, P.J., Field, A.S., Burdette, J.H., Maldjian, J.A., Yen, Y.F., Moody, D.M. Dietary caffeine consumption modulates fMRI measures. Neuroimage 2002;17(2):751-757.

37. Addicott, M.A., Yang, L.L., Peiffer, A.M., et al. The effect of daily caffeine use on cerebral blood flow: How much caffeine can we tolerate? Hum Brain Mapp 2009;30(10):3102-3114.

38. Honey, G., Bullmore, E. Human pharmacological MRI. Trends Pharmacol Sci. 2004;25(7):366-374. Review. 
39. Laurienti, P.J., Field, A.S., Burdette, J.H., Maldjian, J.A., Yen, Y.F., Moody, D.M. Relationship between caffeine-induced changes in resting cerebral perfusion and blood oxygenation level-dependent signal. AJNR Am J Neuroradiol 2004;24(8):1607-1611.

40. Dockstader, C., Gaetz, W., Cheyne, D., Wang, F., Castellanos, F.X., Tannock, R. MEG event-related desynchronization and synchronization deficits during basic somatosensory processing in individuals with ADHD. Behav Brain Funct. 2008;12:4-8.

41. Helenius, P., Laasonen, M., Hokkanen, L., Paetau, R., Niemivirta, M. Impaired engagement of the ventral attentional pathway in ADHD. Neuropsychologia. 2011;49(7):18891896.

42. Uhlhaas, P.J., Singer, W. Neural synchrony in brain disorders: relevance for cognitive dysfunctions and pathophysiology. Neuron. 2006;52:155-168.

43. Fries, P., Nikolic, D., Singer, W. The gamma cycle. Trends Neurosci 2007;30:309-316.

44. Fries, P. Neuronal gamma-band synchronization as a fundamental process in cortical computation. Annu Rev Neurosci. 2009;32:209-224.

45. Bartos M, Vida I, Jonas P. Synaptic mechanisms of synchronized gamma oscillations in inhibitory interneuron networks. Nat Rev Neurosci. 2007;8:45-56.

46. Wilson, T.W., Heinrichs-Graham, E., White, M.L., Knott, N.L., Wetzel, M.W. Estimating the passage of minutes: Deviant oscillatory frontal activity in medicated and un-medicated ADHD. Neuropsychology 2013;27(6):654-665.

47. Wilson, T.W., Wetzel, M.W., White, M.L., Knott, N.L. Gamma-frequency neuronal activity is diminished in adults with attention-deficit/hyperactivity disorder: A pharmaco-MEG study. $J$ Psychopharmacol 2012;26(6):771-777.

48. Franzen, J.D., Wilson, T.W. Amphetamines modulate prefrontal $\gamma$ oscillations during attention processing. Neuroreport 2012;23(12):731-735.

49. Heinrichs-Graham, E., Franzen, J.D., Knott, N.L., White, M.L., Wetzel, M.W., Wilson, T.W. Pharmaco-MEG evidence for attention related hyper-connectivity between auditory and prefrontal cortices in ADHD. Psychiatry Res. 2014;221(3):240-245.

50. ter Huurne, N., Onnink, M., Kan, C., Franke, B., Buitelaar, J., Jensen, O. Behavioral consequences of aberrant alpha lateralization in attention-deficit/hyperactivity disorder. Biol Psychiatry. 2013;74(3):227-233.

51. Wilson, T.W., Franzen, J.D., Heinrichs-Graham, E., White, M.L., Knott, N.L., Wetzel, M.W. Broadband neurophysiological abnormalities in the medial prefrontal region of the default-mode network in adults with ADHD. Hum Brain Mapp. 2013;34(3):566-574. 
52. Franzen, J.D., Heinrichs-Graham, E., White, M.L., Wetzel, M.W., Knott, N.L., Wilson, T.W. Atypical coupling between posterior regions of the default-mode network in attentiondeficit/hyperactivity disorder: A pharmaco-MEG study. J Psychiatry Neurosci. 2013;38(5):333340 .

53. American Psychiatric Association. Diagnostic and statistical manual of mental disorders. $5^{\text {th }}$ ed. Arlington, VA: American Psychiatric Publishing; 2013.

54. Kessler, R.C., Chiu, W.T., Demler, O., et al. Prevalence, severity, and comorbidity of 12month DSM-IV disorders in the National Comorbidity Survey Replication. Archives of General Psychiatry 2005;62:617-622.

55. Kilpatrick, D.G., Resnick, H.S., Milanik, M.E., et al. National estimates of exposure to traumatic events and PTSD prevalence using DSM-IV and DSM-5 criteria. Journal of Traumatic Stress 2013;26(5):537-547.

56. Seal, K., Bertenthal, D., Miner, C., et al. Bringing the War Back Home: Mental Health Disorders Among 103,788 US Veterans Returning From Iraq and Afghanistan Seen at Department of Veterans Affairs Facilities. Archives of Internal Medicine 2007;167:476-482.

57. Seal, K., Metzler, T., Gima, K., et al. Trends and Risk Factors for Mental Health Diagnoses Among Iraq and Afghanistan Veterans Using Department of Veterans Affairs Health Care, 2002-2008. American Journal of Public Health 2009;99(9):1651-1658.

58. Engdahl, B., Leuthold, A.C., Tan, H-R.M., Lewis, S.M., Winskowski, A.M., Dikel, T.N., Georgopoulos, A.P. Post-traumatic stress disorder: a right temporal lobe syndrome? J Neural Eng. 2010;7(6):066005.

59. Georgopoulos, A.P., Tan H-R.M., Lewis, S.M., Leuthold, A.C., Winskowski, A.M., Lynch, J.K., Engdahl, B. The synchronous neural interactions test as a functional neuromarker for post-traumatic stress disorder (PTSD): a robust classification method based on the bootstrap. J Neural Eng. 2010;7(1):16011.

60. James, L.M., Belitskaya-Lévy, I., Lu, Y., Wang, H., Engdahl, B.E., Leuthold, A.C., Georgopoulos, A.P. Development and application of a diagnostic algorithm for posttraumatic stress disorder. Psychiatry Res. 2015;231(1):1-7.

61. Anders, S.L., Peterson, C.K., James, L.M., Engdahl, B., Leuthold, A.C., Georgopoulos, A.P. Neural communication in posttraumatic growth. Exp Brain Res. 2015;233(7):2013-2020.

62. James, L.M., Engdahl, B.E., Leuthold, A.C., Lewis, S.M., Van Kampen, E., Georgopoulos, A.P. Neural network modulation by trauma as a marker of resilience: differences between veterans with posttraumatic stress disorder and resilient controls. JAMA Psychiatry. 2013;70(4):410-418. 
63. Kolassa, I.T., Wienbruch, C., Neuner, F., Schauer, M., Ruf, M., Odenwald, M., Elbert, T. Altered oscillatory brain dynamics after repeated traumatic stress. BMC Psychiatry. 2007;7:56.

64. Adenauer, H., Pinösch, S., Catani, C., Gola, H., Keil, J., Kissler, J., Neuner, F. Early processing of threat cues in posttraumatic stress disorder-evidence for a cortical vigilanceavoidance reaction. Biol Psychiatry. 2010;68(5):451-458.

65. Todd, R.M., MacDonald, M.J., Sedge, P., Robertson, A., Jetly, R., Taylor, M.J., Pang, E.W. Soldiers with Posttraumatic Stress Disorder See a World Full of Threat:

Magnetoencephalography Reveals Enhanced Tuning to Combat-Related Cues. Biol Psychiatry. 2015 May 27.

66. Badura-Brack, A.S., Becker, K.M., McDermott, T.J., Ryan, T.J., Becker, M.M., Hearley, A.R., Heinrichs-Graham, E., Wilson, T.W. Decreased somatosensory activity to non-threatening touch in combat veterans with posttraumatic stress disorder. Psychiatry Res. 2015;233(2):194-200.

67. McDermott, T.J., Badura-Brack, A.S., Becker, K.M., Ryan, T.J., Khanna, M.M., HeinrichsGraham, E., \& Wilson, T.W. Male veterans with PTSD exhibit aberrant neural dynamics during working memory encoding: A MEG study. Journal of Psychiatry and Neuroscience. (in press)

68. McDermott, T.J., Badura-Brack, A.S., Becker, K.M., Ryan, T.J., Bar-Haim, Y., Pine, D.S., Abend, R., Khanna, M.M., Heinrichs-Graham, E., \& Wilson, T.W. Attention training improves aberrant neural dynamics during working memory encoding in male veterans with PTSD. Psychological Medicine.

69. Dunkley, B.T., Doesburg, S.M., Sedge, P.A., Grodecki, R.J., Shek, P.N., Pang, E.W., Taylor, M.J. Resting-state hippocampal connectivity correlates with symptom severity in post-traumatic stress disorder. Neuroimage Clin. 2014;5:377-384.

70. Dunkley, B.T., Sedge, P.A., Doesburg, S.M., Grodecki, R.J., Jetly, R., Shek, P.N., Taylor, M.J., Pang, E.W. Theta, mental flexibility, and post-traumatic stress disorder: connecting in the parietal cortex. PLoS One. 2015;10(4):e0123541.

71. Dunkley, B.T., Doesburg, S.M., Jetly, R., Sedge, P.A., Pang, E.W., Taylor, M.J. Characterising intra- and inter-intrinsic network synchrony in combat-related post-traumatic stress disorder. Psychiatry Res. 2015.

72. Antiretroviral Therapy Cohort Collaboration. Life expectancy of individuals on combination antiretroviral therapy in high-income countries: a collaborative analysis of 14 cohort studies. Lancet. 2008;372:293-299.

73. Nakagawa, F., May, M., Phillips, A. Life expectancy living with HIV: recent estimates and future implications. Curr Opin Infect Dis. 2016;26:17-25. 
74. Antinori, A., Arendt, G., Becker, J.T., Brew, B.J., Byrd, D.A., Cherner, M., et al. Updated research nosology for HIV-associated neurocognitive disorders. Neurology. 2007;69:1789-1799.

75. Cysique, L.A., Brew, B.J. Neuropsychological functioning and antiretroviral treatment in HIV/AIDS: A review. [Review]. Neuropsychol Rev. 2009;19:169-185.

76. Gannon, P., Khan, M.Z., Kolson, D.L. Current understanding of HIV-associated neurocognitive disorders pathogenesis. Curr Opin Neurol. 2011;24:275-283. Review.

77. Heaton, R.K., Clifford, D.B., Franklin, D.R. Jr., Woods, S.P., Ake, C., Vaida, F., et al. HIVassociated neurocognitive disorders persist in the era of potent antiretroviral therapy: CHARTER Study. Neurology. 2010;75:2087-2096.

78. Heaton, R.K., Franklin, D.R., Ellis, R.J., McCutchan, J.A., Letendre, S.L., Leblanc, S., et al. HIV-associated neurocognitive disorders before and during the era of combination antiretroviral therapy: differences in rates, nature, and predictors. J Neurovirol. 2001;17:3-16.

79. Robertson, K.R., Smurzynski, M., Parsons, T.D., Wu, K., Bosch, R.J., Wu, J., et al. The prevalence and incidence of neurocognitive impairment in the HAART era. AIDS. 2007;21:1915-1921.

80. Simioni, S., Cavassini, M., Annoni, J.M., Rimbault Abraham, A., Bourquin, I., Schiffer, V., et al. Cognitive dysfunction in HIV patients despite long-standing suppression of viremia. AIDS. 2010;24:1243-1250.

81. Tozzi, V., Balestra, P., Bellagamba, R., Corpolongo, A., Salvatori, M.F., Visco-Comandini, U., et al. Persistence of neuropsychologic deficits despite long-term highly active antiretroviral therapy in patients with HIV-related neurocognitive impairment: Prevalence and risk factors. $J$ Acquir Immune Defic Syndr. 2007;45:174-182.

82. Wilson, T.W., Heinrichs-Graham, E., Robertson, K.R., Sandkovsky, U., O'Neill, J., Knott, N.L., Fox, H.S., Swindells, S. Functional brain abnormalities during finger-tapping in HIV-infected older adults: a magnetoencephalography study. J Neuroimmune Pharmacol. 2013;8(4):965-74.

83. Wilson, T.W., Fox, H.S., Robertson, K.R., Sandkovsky, U., O'Neill, J., Heinrichs-Graham, E., Knott, N.L., Swindells, S. Abnormal MEG oscillatory activity during visual processing in the prefrontal cortices and frontal eye-fields of the aging HIV brain. PLoS One. 2013;8(6):e66241.

84. Becker, J.T., Bajo, R., Fabrizio, M., Sudre, G., Cuesta, P., Aizenstein, H.J., et al. Functional connectivity measured with magnetoencephalography identifies persons with HIV disease. Brain Imaging Behav. 2012;6:366-373. 
85. Becker, K.M., Heinrichs-Graham, E., Fox, H.S., Robertson, K.R., Sandkovsky, U., O'Neill, J., Swindells, S., Wilson, T.W. Decreased MEG beta oscillations in HIV-infected older adults during the resting state. J Neurovirol. 2013;19(6):586-594.

86. Wilson, T.W., Heinrichs-Graham, E., Becker, K.M., Aloi, J., Robertson, K.R., Sandkovsky, U., White, M.L., O'Neill, J., Knott, N.L., Fox, H.S., Swindells, S. Multimodal neuroimaging evidence of alterations in cortical structure and function in HIV-infected older adults. Hum Brain Mapp. 2015;36(3):897-910.

87. Becker, J.T., Fabrizio, M., Sudre, G., Haridis, A., Ambrose, T., Aizenstein, H.J., et al. Potential utility of resting-state magnetoencephalography as a biomarker of CNS abnormality in HIV disease. J Neurosci Methods. 2012;206:176-182.

88. Schrag, A., Ben-Shlomo, Y., and Quinn, N. How valid is the clinical diagnosis of Parkinson's disease in the community? J Neurol Neurosurgy Psychiatry. 2002;73:529-534.

89. Wright Willis, A., Evanoff, B.A., Lian, M., Criswell, S.R., and Racette, B.A. Geographic and ethnic variation in Parkinson disease: a population-based study of US Medicare beneficiaries. Neuroepidemiology. 2012;34:143-151.

90. Dorsey, E.R., Constantinescu, R., Thompson, J.P., et al. Projected number of people with Parkinson disease in the most populous nations, 2005 through 2030. Neurology. 2007;68:384386.

91. Bartels, A.L., Balash, Y., Gurevich, T., Schaafsma, J.D., Hausdorff, J.M., and Giladi, N. Relationship between freezing of gait (FOG) and other features of Parkinson's: FOG is not correlated with bradykinesia. J Clin Neurosci. 2003;10:584-588.

92. Dewey, R.B., Jr., Taneja, A., McClintock, S.M., Cullum, C.M., Dewey, R.B. 3rd, Bernstein, I., and Husain, M.M. Motor symptoms at onset of Parkinson disease and risk for cognitive impairment and depression. Cogn Behav Neurol. 2012;25:115-120.

93. Fahn, S. The history of dopamine and levodopa in the treatment of Parkinson's disease. Mov Disord. 2008;23 Suppl 3:S497-508.

94. Fahn, S., Oakes, D., Shoulson, I., Kieburtz, K., Rudolph, A., Lang, A., Olanow, C.W., Tanner, C., Marek, K., and Parkinson Study, G. Levodopa and the progression of Parkinson's disease. New Engl J Med. 2004;351:2498-2508.

95. Jankovic, J. Parkinson's disease: clinical features and diagnosis. J Neurol Neurosurg Psychiatry. 2008;79:368-376.

96. Damier, P., Hirsch, E.C., Agid, Y., and Graybiel, A.M. The substantia nigra of the human brain. II. Patterns of loss of dopamine-containing neurons in Parkinson's disease. Brain. 1999;122(8):1437-1448. 
97. Litvak, V., Jha, A., Eusebio, A., Oostenveld, R., Foltynie, T., Limousin, P., Zrinzo, L., Hariz, M.I., Friston, K., and Brown, P. Resting oscillatory cortico-subthalamic connectivity in patients with Parkinson's disease. Brain. 2011;134:359-374.

98. Litvak, V., Eusebio, A., Jha, A., et al. Movement-related changes in local and long-range synchronization in Parkinson's disease revealed by simultaneous magnetoencephalography and intracranial recordings. J Neurosci. 2012;32:10541-10553.

99. Hirschmann, J., Ozkurt, T.E., Butz, M., Homburger, M., Elben, S., Hartmann, C.J., Vesper, J., Wojtecki, L., and Schnitzler, A. Distinct oscillatory STN-cortical loops revealed by simultaneous MEG and local field potential recordings in patients with Parkinson's disease. NeuroImage. 2011;55:1159-1168.

100. Hirschmann, J., Hartmann, C.J., Butz, M., Hoogenboom, N., Ozkurt, T.E., Elben, S., Vesper, J., Wojtecki, L., and Schnitzler, A. A direct relationship between oscillatory subthalamic nucleus-cortex coupling and rest tremor in Parkinson's disease. Brain. 2013;136:3659-3670.

101. Bock, A., Kuhn, A.A., Trahms, L., and Sander, T.H. Validity of subthalamic-cortical coherency observed in patients with Parkinson's disease. Biomed Engin. 2013;58:157-164.

102. Hirschmann, J., Ozkurt, T.E., Butz, M., Homburger, M., Elben, S., Hartmann, C.J., Vesper, J., Wojtecki, L., and Schnitzler, A. (2013). Differential modulation of STN-cortical and corticomuscular coherence by movement and levodopa in Parkinson's disease. NeuroImage.

2013;68:203-213.

103. Brown, P., Williams, D. Basal ganglia local field potential activity: character and functional significance in the human. Clin Neurophysiol. 2005;116:2510-2519.

104. Hutchison, W.D., Dostrovsky, J.O., Walters, J.R., Courtemanche, R., Boraud, T., Goldberg, J., and Brown, P. Neuronal oscillations in the basal ganglia and movement disorders: evidence from whole animal and human recordings. J Neurosci. 2004;24:9240-9243.

105. Salenius, S., Avikainen, S., Kaakkola, S., Hari, R., and Brown, P. Defective cortical drive to muscle in Parkinson's disease and its improvement with levodopa. Brain. 2002;125, 491-500.

106. Pollok, B., Kamp, D., Butz, M., Wojtecki, L., Timmermann, L., Sudmeyer, M., Krause, V., and Schnitzler, A. Increased SMA-M1 coherence in Parkinson's disease - Pathophysiology or compensation? Exp Neurol. 2013;247:178-181.

107. Pollok, B., Makhloufi, H., Butz, M., Gross, J., Timmermann, L., Wojtecki, L., and Schnitzler, A. Levodopa affects functional brain networks in Parkinsonian resting tremor. Mov Disord 2009;24:91-98.

108. Timmermann, L., Gross, J., Dirks, M., Volkmann, J., Freund, H.J., and Schnitzler, A. The cerebral oscillatory network of parkinsonian resting tremor. Brain. 2003;126:199-212. 
109. Bosboom, J.L., Stoffers, D., Stam, C.J., van Dijk, B.W., Verbunt, J., Berendse, H.W., and Wolters, E. Resting state oscillatory brain dynamics in Parkinson's disease: an MEG study. Clin Neurophysiol. 2006:117:2521-2531.

110. Stoffers, D., Bosboom, J.L., Deijen, J.B., Wolters, E., Stam, C.J., and Berendse, H.W. Increased cortico-cortical functional connectivity in early-stage Parkinson's disease: an MEG study. NeuroImage. 2008;41:212-222.

111. Stoffers, D., Bosboom, J.L., Deijen, J.B., Wolters, E.C., Berendse, H.W., and Stam, C.J. Slowing of oscillatory brain activity is a stable characteristic of Parkinson's disease without dementia. Brain. 2007;130:1847-1860.

112. Heinrichs-Graham, E., Kurz, M.J., Becker, K.M., Santamaria, P.M., Gendelman, H.E., and Wilson, T.W. Hypersynchrony despite pathologically reduced beta oscillations in patients with Parkinson's disease: a pharmaco-magnetoencephalography study. J Neurophysiol. 2014;112:1739-1747.

113. Heinrichs-Graham, E., Wilson, T.W., Santamaria, P.M., Heithoff, S.K., Torres-Russotto, D., Hutter-Saunders, J.A., Estes, K.A., Meza, J.L., Mosley, R.L., and Gendelman, H.E. Neuromagnetic evidence of abnormal movement-related beta desynchronization in Parkinson's disease. Cereb Cortex. 2014;24:2669-2678.

114. Gaetz, W., Macdonald, M., Cheyne, D., and Snead, O.C. Neuromagnetic imaging of movement-related cortical oscillations in children and adults: age predicts post-movement beta rebound. NeuroImage. 2010;51:792-807.

115. Cheyne, D., Bakhtazad, L., and Gaetz, W. Spatiotemporal mapping of cortical activity accompanying voluntary movements using an event-related beamforming approach. Hum Brain Mapp. 2006;27:213-229.

116. Jurkiewicz, M.T., Gaetz, W.C., Bostan, A.C., and Cheyne, D. Post-movement beta rebound is generated in motor cortex: evidence from neuromagnetic recordings. NeuroImage. 2006;32:1281-1289.

117. Wilson, T.W., Slason, E., Asherin, R., Kronberg, E., Teale, P.D., Reite, M.L., and Rojas, D.C. Abnormal gamma and beta MEG activity during finger movements in early-onset psychosis. Dev Neuropsychol. 2011;36:596-613.

118. Wilson, T.W., Slason, E., Asherin, R., Kronberg, E., Reite, M.L., Teale, P.D., and Rojas, D.C. An extended motor network generates beta and gamma oscillatory perturbations during development. Brain Cogn. 2010;73:75-84.

119. Heinrichs-Graham, E., Wilson, T.W. Coding complexity in the human motor circuit. Hum Brain Mapp. 2015 Sep 25. doi: 10.1002/hbm.23000. [Epub ahead of print] . 
120. Wilson, T.W., Heinrichs-Graham, E., Becker, K.M. Circadian modulation of motor-related beta oscillatory responses. Neuroimage. 2014;102(2):531-539.

121. Wilson, T.W., Fleischer, A., Archer, D., Hayasaka, S., Sawaki, L. Oscillatory MEG motor activity reflects therapy-related plasticity in stroke patients. Neurorehabil Neural Repair. 2011;25(2):188-193.

122. te Woerd, E.S., Oostenveld, R., de Lange, F.P., and Praamstra, P. A shift from prospective to reactive modulation of beta-band oscillations in Parkinson's disease. NeuroImage. 2014;100:507-519.

123. te Woerd, E.S., Oostenveld, R., Bloem, B.R., de Lange, F.P., and Praamstra, P. (2015). Effects of rhythmic stimulus presentation on oscillatory brain activity: the physiology of cueing in Parkinson's disease. NeuroImage Clin. 2015;9:300-309.

124. Christensen, D., Van Naarden, B.K., Doernberg, N.S., Maenner, M.J., Arneson, C.L., Durkin, M.S., Benedict, R.E., Kirby, R.S., Wingate, M.S., Fitzgerald, R., Yeargin-Allsopp, M. Prevalence of cerebral palsy, co-occurring autism spectrum disorders, and motor functioning Autism and Developmental Disabilities Monitoring Network, USA, 2008. Dev Med Child Neurol. 2014;56:59-65.

125. Yeargin-Allsopp, M., Van Naarden B.K., Doernberg, N.S., Benedict, R.E., Kirby, K.S., Durkin, M.S. Prevalence of cerebral palsy in 8-year-old children in three areas of the United States in 2002:a multisite collaboration. Pediatrics. 2008;121(3):547-554.

126. Center for Disease Control and Prevention. Economic costs associated with mental retardation, cerebral palsy, hearing loss, and vision impairment--United States, 2003. MMWR 2004:53:57-59.

127. Kurz, M.J., Wilson, T.W. Neuromagnetic activity in the somatosensory cortices of children with cerebral palsy. Neurosci Lett. 2011;490(1):1-5.

128. Kurz, M.J., Wilson, T.W., Corr, B., Volkman, K.G. Neuromagnetic activity of the somatosensory cortices associated with body weight-supported treadmill training in children with cerebral palsy. J Neurol Phys Ther. 2012;36(4):166-172.

129. Kurz, M.J., Heinrichs-Graham, E., Arpin, D.J., Becker, K.M., Wilson, T.W. Aberrant synchrony in the somatosensory cortices predicts motor performance errors in children with cerebral palsy. J Neurophysiol. 2014;111(3):573-579.

130. Kurz, M.J., Heinrichs-Graham, E., Becker, K.M., Wilson, T.W. The magnitude of the somatosensory cortical activity is related to the mobility and strength impairments seen in children with cerebral palsy. J Neurophysiol. 2015;113(9):3143-3150. 
131. Kurz, M.J., Becker, K.M., Heinrichs-Graham, E., Wilson, T.W. Children with cerebral palsy have uncharacteristic somatosensory cortical oscillations after stimulation of the hand mechanoreceptors. Neuroscience. 2015;305:67-75.

132. Kurz, M.J., Becker, K.M., Heinrichs-Graham, E., Wilson, T.W. Neurophysiological abnormalities in the sensorimotor cortices during the motor planning and movement execution stages of children with cerebral palsy. Dev Med Child Neurol. 2014;56(11):1072-1077.

133. Valvano J. Neurophysiological evidence for motor planning limitations in children with cerebral palsy. Dev Med Child Neurol. 2014;56(11):1035-1036.

134. Salthouse, T.A., Babcock, R.L., Shaw, R.J. Effects of adult age on structural and operational capacities in working memory. Psychol Aging. 1991;6:118-127.

135. Park, D.C., et al. Models of visuospatial and verbal memory across the adult life span. Psychol Aging. 2002;17:299-320.

136. Reuter-Lorenz, P.A., Cappell, K. A. Neurocognitive aging and the compensation hypothesis. Curr Dir Psychol Sci. 2008;17:177-182.

137. Park, J. et al. Neural broadening or neural attenuation? Investigating age-related dedifferentiation in the face network in a large lifespan sample. $J$ Neurosci. 2012;32:2154-2158.

138. Alain, C., McDonald, K.L. Age-related differences in neuromagnetic brain activity underlying concurrent sound perception. J Neurosci. 2007;27:1308-1314.

139. Matilainen, L.E., Talvitie, S.S., Pekkonen, E., Alku, P., May, P.J., Tiitinen, H. The effects of healthy aging on auditory processing in humans as indexed by transient brain responses. Clin Neurophysiol. 2010;121(6):902-911.

140. Cheng, C.H., Baillet, S., Hsiao, F.J., Lin, Y.Y. Effects of aging on the neuromagnetic mismatch detection to speech sounds. Biol Psychol. 2015;104:48-55.

141. Aine, C.J., Adair, J.C., Knoefel, J.E., et al. Temporal dynamics of age-related differences in auditory incidental verbal learning. Cogn Brain Res. 2005;24(1):1-18.

142. Kovacevic, S., Qualls, C., Adair, J.C., et al. Age-related effects on superior temporal gyrus activity during an auditory oddball task. Neuroreport. 2005;16(10):1075-1079.

143. Cheng, C.H., Lin, Y.Y. The effects of aging on lifetime of auditory sensory memory in humans. Biol Psychol. 2012;89(2):306-312.

144. Ross, B., Fujioka, T., Tremblay, K.L., Picton, T.W. Aging in binaural hearing begins in mid-life: evidence from cortical auditory-evoked responses to changes in interaural phase. $J$ Neurosci. 2007;27(42):11172-11178. 
145. Ross, B., Snyder, J.S., Aalto, M., et al. Neural encoding of sound duration persists in older adults. Neuroimage. 2009;47(2):678-687.

146. Huttunen, J., Wikstrom, H., Salonen, O., Ilmoniemi, R.J. Human somatosensory cortical activation strengths: comparison between males and females and age-related changes. Brain Res. 1999;818(2):196-203.

147. Stephen, J.M., Ranken, D., Best, E., et al. Aging changes and gender differences in response to median nerve stimulation measured with MEG. Clin Neurophysiol. 2006;117(1):131-143.

148. Hagiwara, K., Ogata, K., Okamoto, T., et al. Age-related changes across the primary and secondary somatosensory areas: an analysis of neuromagnetic oscillatory activities. Clin Neurophysiol. 2014;125(5):1021-1029.

149. Zappasodi, F., Pasqualetti, P., Tombini, M., et al. Hand cortical representation at rest and during activation: gender and age effects in the two hemispheres. Clin Neurophysiol. 2006;117(7):1518-1528.

150. Goto, S., Fujisawa, Y., Uemura, J., Yamada, S., Hoshiyama, M., Hirayama, M. Disinhibitory shift of recovery curve of somatosensory-evoked response in elderly: A magnetoencephalographic study. Clin Neurophysiol. 2015;126(6):1228-1233.

151. Cheng, C.H., Chan, P.Y., Baillet, S., Lin, Y.Y. Age-Related Reduced Somatosensory Gating Is Associated with Altered Alpha Frequency Desynchronization. Neural Plast. 2015;2015:302878.

152. Vlahou, E.L., Thurm, F., Kolassa, I.T., Schlee, W. Resting-state slow wave power, healthy aging and cognitive performance. Sci Rep. 2014;4:5101.

153. Leirer, V.M., Wienbruch, C., Kolassa, S., Schlee, W., Elbert, T., Kolassa, I.T. Changes in cortical slow wave activity in healthy aging. Brain Imaging Behav. 2011;5(3):222-228.

154. Schlee, W., Leirer, V., Kolassa, S., Thurm, F., Elbert, T., Kolassa, I.T. Development of large-scale functional networks over the lifespan. Neurobiol Aging. 2012;33(10):2411-2421.

155. Schlee, W., Leirer, V., Kolassa, I.T., Weisz, N., Elbert, T. Age-related changes in neural functional connectivity and its behavioral relevance. BMC Neurosci. 2012;13:16.

156. Proskovec, A.L., Heinrichs-Graham, E. \& Wilson, T.W. Aging modulates the oscillatory dynamics underlying successful working memory encoding and maintenance. Hum Brain Mapp.

157. Aine, C.J., Woodruff, C.C., Knoefel, J.E., et al. Aging: compensation or maturation? NeuroImage. 2006;32(4):1891-1904.

158. Bonnefond, M., Jensen, O. Alpha oscillations serve to protect working memory maintenance against anticipated distracters. Current Biology. 2012;22:1969-1974. 
159. Jiang, H., van Gerven, M.A., Jensen, O. Modality-specific Alpha Modulations Facilitate Long-term Memory Encoding in the Presence of Distracters. J Cogn Neurosci. 2015;27:583-592.

160. Tuladhar, A.M., ter Huurne, N., Schoffelen, J.M., Maris, E., Oostenveld, R., Jensen, O. Parieto-occipital sources account for the increase in alpha activity with working memory load. Human Brain Mapp. 2007;28:785-792.

161. Alem, O., Benison, A.M., Barth, D.S., Kitching, J., Knappe, S. Magnetoencephalography of epilepsy with a microfabricated atomic magnetrode. J Neurosci. 2014;34(43):14324-14327. 


\section{Figure Captions}

Figure 1: Typical Magnetoencephalography (MEG) System. (A) A modern whole-head MEG system equipped with 306 cortical sensors. MEG instruments are housed in magneticallyshielded rooms to reduce interference from the environment's ambient magnetic fields. (B) Encased within the MEG helmet is a large array of magnetic sensors that are immersed in liquid helium. This helium endows the sensor array, and associated elements, with superconducting properties which are needed to measure the ultra-minute magnetic fields, but also creates a significant maintenance cost as the Helium has to be replenished periodically. In (B), the array of the 306-sensor instrument shown in (A) is depicted. Each square represents a sensor chip (102 total) and is comprised of 3 independent MEG sensors, with each sensor having a different sensitivity to components of the magnetic fields.

Figure 2. Number of MEG Publications per Year. Since David Cohen published the first MEG article in 1968, the number of MEG publications per year has steadily increased, rising sharply over the past 15 to 20 years. This figure is based on a PubMed search conducted in early 2016 using the term "magnetoencephalography.”

Figure 3. MEG Oscillations and Dynamics. (A) Example output from a MEG source reconstruction, using a beamforming algorithm, of beta oscillatory activity during the planning stage of a right-hand movement $(-0.45 \mathrm{~s}$ to $0.0 \mathrm{~s} ; 0.0 \mathrm{~s}=$ movement onset $)$ in healthy individuals. To more precisely examine the dynamics, “virtual sensors” can be extracted from any voxel in the brain and these data have the same temporal precision as the original recording (e.g., $1 \mathrm{~ms}$ ). In this example, virtual sensors were extracted from the peak voxel in each hemisphere, which 
corresponded to the bilateral primary motor cortices (yellow dots). (B) Time-frequency spectrograms of oscillatory power (relative to baseline) for the voxels extracted from left and right primary motor cortices. For each spectrogram, frequency (in $\mathrm{Hz}$ ) is shown on the y-axis, while time (in s) is shown on the x-axis. Color bars reflect positive (red) and negative (blue) values relative to the baseline period, which was -2.0 to $-1.2 \mathrm{~s}$ before motor onset (movement onset $=0.0 \mathrm{~s}$ ). Negative activity represents an event-related desynchronization (ERD), whereas the positive activity constitutes an event-related synchronization (ERS). (C) Time course of beta activity (16-26 Hz) for each peak voxel. For each voxel time series, relative power (in percentage from baseline) is shown on the y-axis, while time (in s) is shown on the x-axis. Error bars (shaded) reflect standard error of the mean (SEM).

\section{Figure 4. Functional Connectivity in the Default-Mode Network of Adults with and without}

ADHD. Three models of the default-mode network (DMN) showing group differences and similarities in functional connectivity along particular pathways at distinct frequencies of neuronal activity are shown. See Figure Legend (upper right) for definition of pathway colors. In the ultra-low frequency range, adults without ADHD showed stronger coupling between the medial prefrontal cortices (MPFC) and posterior cingulate cortices (PCC) and the PCC and right inferior parietal (RIPL) areas, whereas those with ADHD showed stronger connectivity between the RIPL and left inferior parietal (LIPL) nodes. In the delta band, controls showed increased connectivity between the MPFC and LIPL regions and the PCC and RIPL regions relative to unmedicated patients with ADHD. Theta frequency neuronal activity was more strongly coupled between the PCC and the RIPL and LIPL areas in adults without ADHD relative to their unmedicated peers with ADHD, while the latter group showed stronger coupling between the RIPL 
and LIPL cortical areas. Interestingly, the increased functional connectivity in the PCC-RIPL pathway of adults without ADHD was consistent throughout all three frequency ranges of neuronal coupling. Following medication administration, all differences in connectivity between adults with and without ADHD dissipated and were no longer significant, indicating that stimulants increased connectivity along some pathways and decreased it along others. ${ }^{52}$

\section{Figure 5. Dynamics of Working Memory Dysfunction in Posttraumatic Stress Disorder} (PTSD). Group mean functional images (beamformer, pseudo-t scale) for controls and veterans with PTSD are displayed in the sagittal orientation for select time windows of the encoding (top) and maintenance (bottom) periods. Note that pseudo-t values reflect the differential power per voxel between an active period (shown below image) and a pre-stimulus baseline period. (Left) Both groups exhibited strong alpha-beta oscillatory responses in the left hemispheric frontotemporal cortices throughout the encoding phase, which narrowed to include only the alpha band during maintenance. Interestingly, there were no group differences in these left fronto-temporal circuits in any time period. (Right) In contrast, veterans with PTSD exhibited significantly stronger oscillatory responses in multiple right hemispheric brain regions during the encoding and maintenance periods relative to demographically-matched controls. The critical regions included the right inferior frontal gyrus (homologue of Broca's area), right supramarginal gyrus, and right temporal cortices $(\mathrm{p}<0.005$, corrected). Note that right hemispheric group differences were observed in other time periods (not shown) as well. ${ }^{67}$

Figure 6. Structural-Functional Deficits in HIV-infected Older Adults. (A) HIV-infected patients had weaker activity in the left postcentral gyrus following tactile stimulation to the right 
hand compared to (B) demographically-matched uninfected participants ( $\mathrm{p}<0.01$, corrected).

(C) The same HIV-infected patients also had reduced gray matter volume relative to uninfected controls in the left postcentral gyrus and several other brain regions (green areas; $\mathrm{p}<0.001$, corrected). This area of reduced gray matter in the left postcentral gyrus spatially overlapped with the maximal MEG response, suggesting that aberrations in brain structure contribute to at least some functional MEG abnormalities in HIV-infected patients. Of note, this study found abnormal MEG responses in the prefrontal cortices of HIV-infected patients as well, and no structural deficits were observed in this region. ${ }^{86}$

Figure 7. Aberrant Beta Oscillations in Parkinson's Disease (PD). (Left) Average timefrequency spectrograms from a MEG sensor near the sensorimotor cortex are shown for healthy controls (top panel) and patients with PD (bottom panel). Time (in ms) is denoted on the x-axis, with 0 ms defined as movement onset. Frequency (in $\mathrm{Hz}$ ) is shown on the $\mathrm{y}$-axis. The typical pattern of peri-movement beta desynchronization (ERD) and post-movement beta rebound (PMBR) during a right-hand movement task, expressed as percent difference from baseline, can be discerned in each group. The time period within the white box (-300 to $200 \mathrm{~ms}$ ) was subjected to source reconstruction analyses. These analyses revealed that peak group differences in the beta ERD response (right) were within the motor hand knob region of the left precentral gyrus and extended onto the adjacent postcentral gyrus $(\mathrm{p}=.035$, corrected). In both cases, responses were weaker in patients with PD. Trending differences were also seen in the right precentral gyrus near the hand knob region ( $\mathrm{p}<.005$, uncorrected; $\mathrm{p}=.107$, corrected) ${ }^{113}$ Abnormalities in beta activity appear to be a central feature of PD and have been observed in the cortex in MEG studies and in the subthalamic nucleus during deep brain stimulation (DBS) surgeries. 


\section{Figure 8. Healthy Aging Modulates the Dynamics of Working Memory Processing. Group} mean beamformer images (pseudo-t) for younger (Left) and older (Middle) adults, with each row depicting a different time period of the early maintenance phase $(0.0 \mathrm{~ms}=$ offset of encoding grid and beginning of memory maintenance). Note that pseudo-t units indicate the differential power per voxel between the active period (far left) and a pre-stimulus baseline period. (Right) Significant group differences $(\mathrm{p}<.005$, corrected) in alpha oscillatory activity in parietooccipital cortices as a function of time. Younger adults showed a shift from a bilateral decrease in alpha activity in the occipital cortices to a right-lateralized increase in alpha activity in parietooccipital areas. Conversely, older adults demonstrated a progressive increase in alpha activity across bilateral occipital cortices, with the increases in alpha occurring earlier and involving more cortical tissue in older adults relative to younger adults. ${ }^{156}$ Such alpha synchronization is thought to decrease or inhibit visual input and thereby aid in maintaining memory representations. 

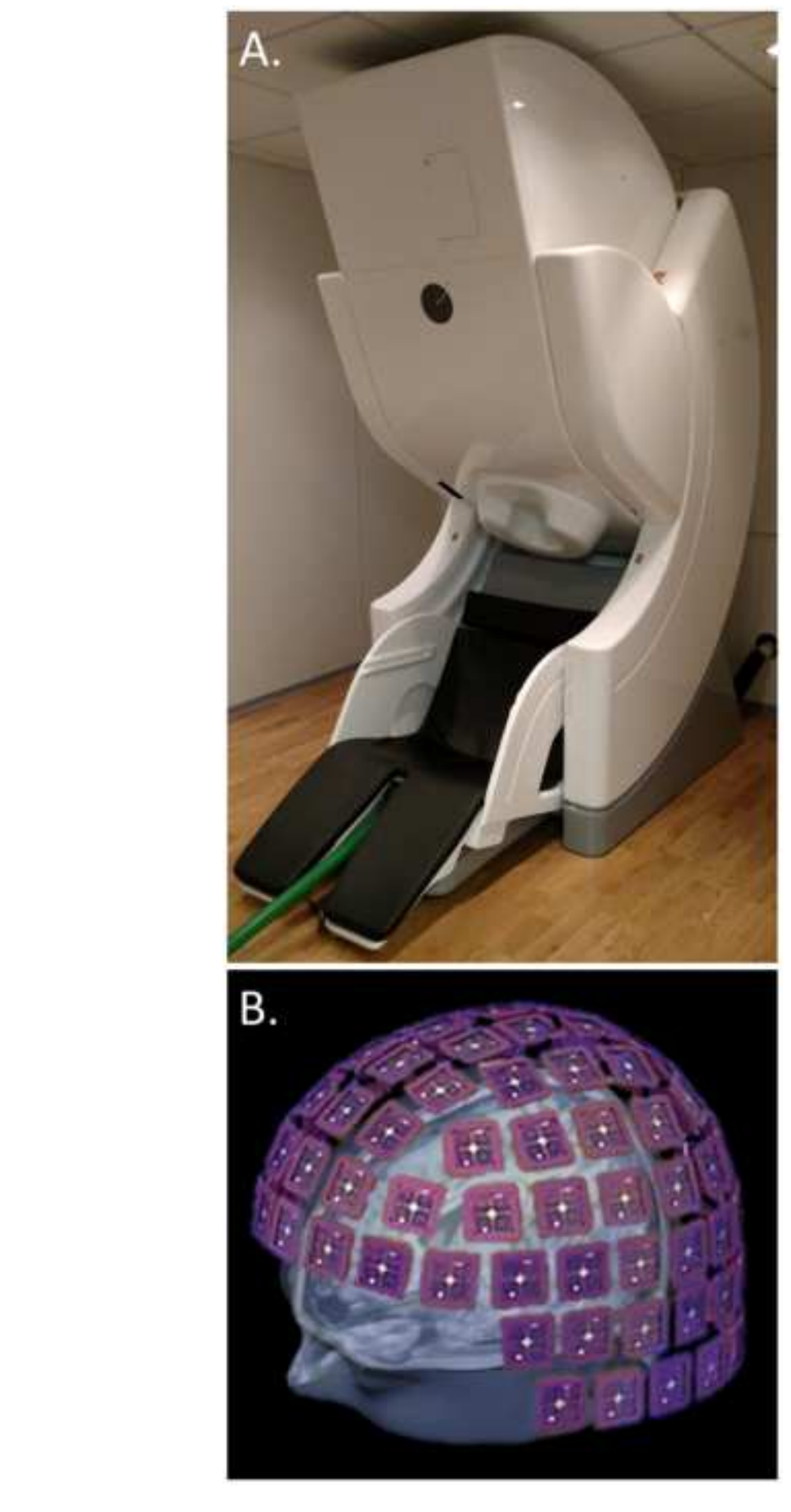

Figure 1

\section{Figure 1}
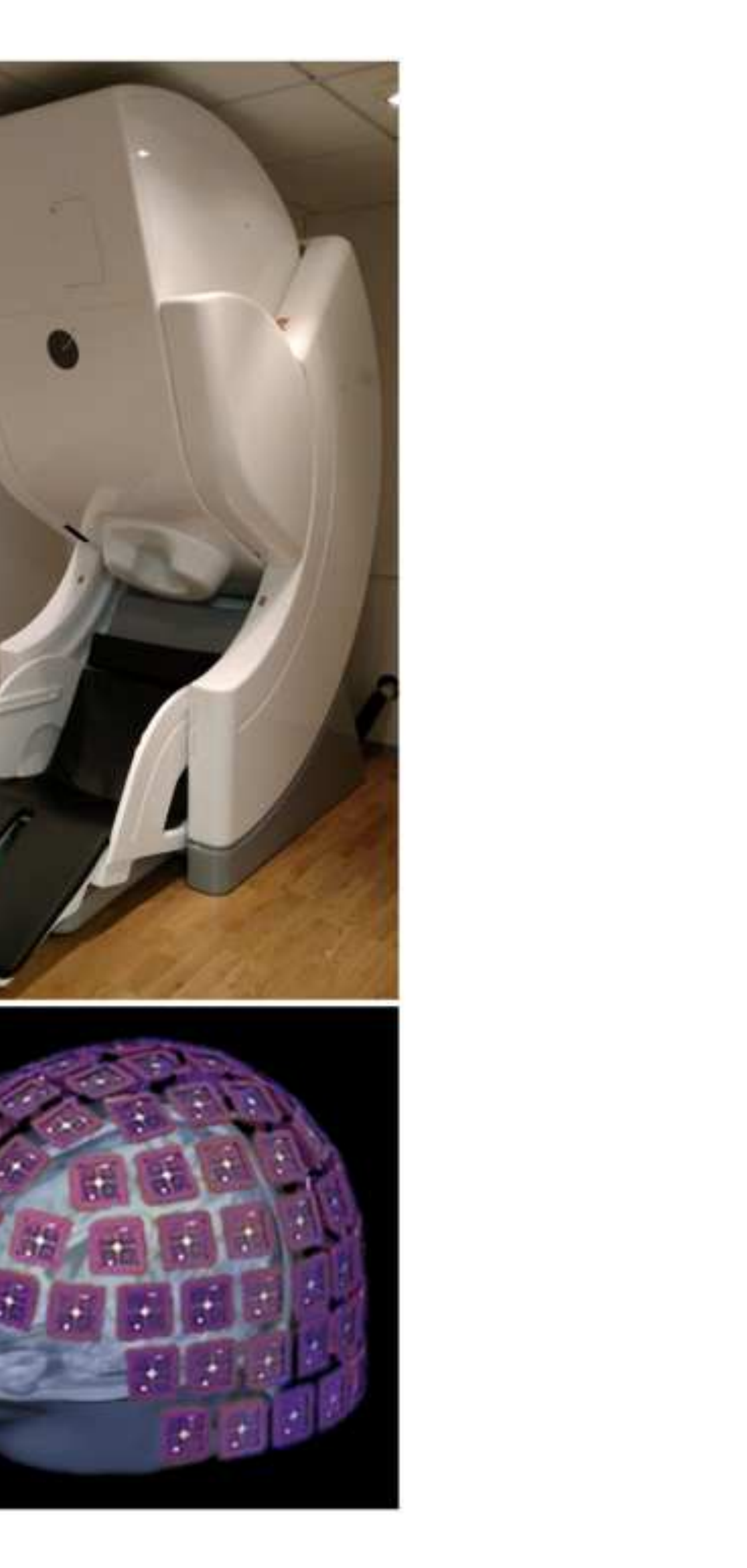


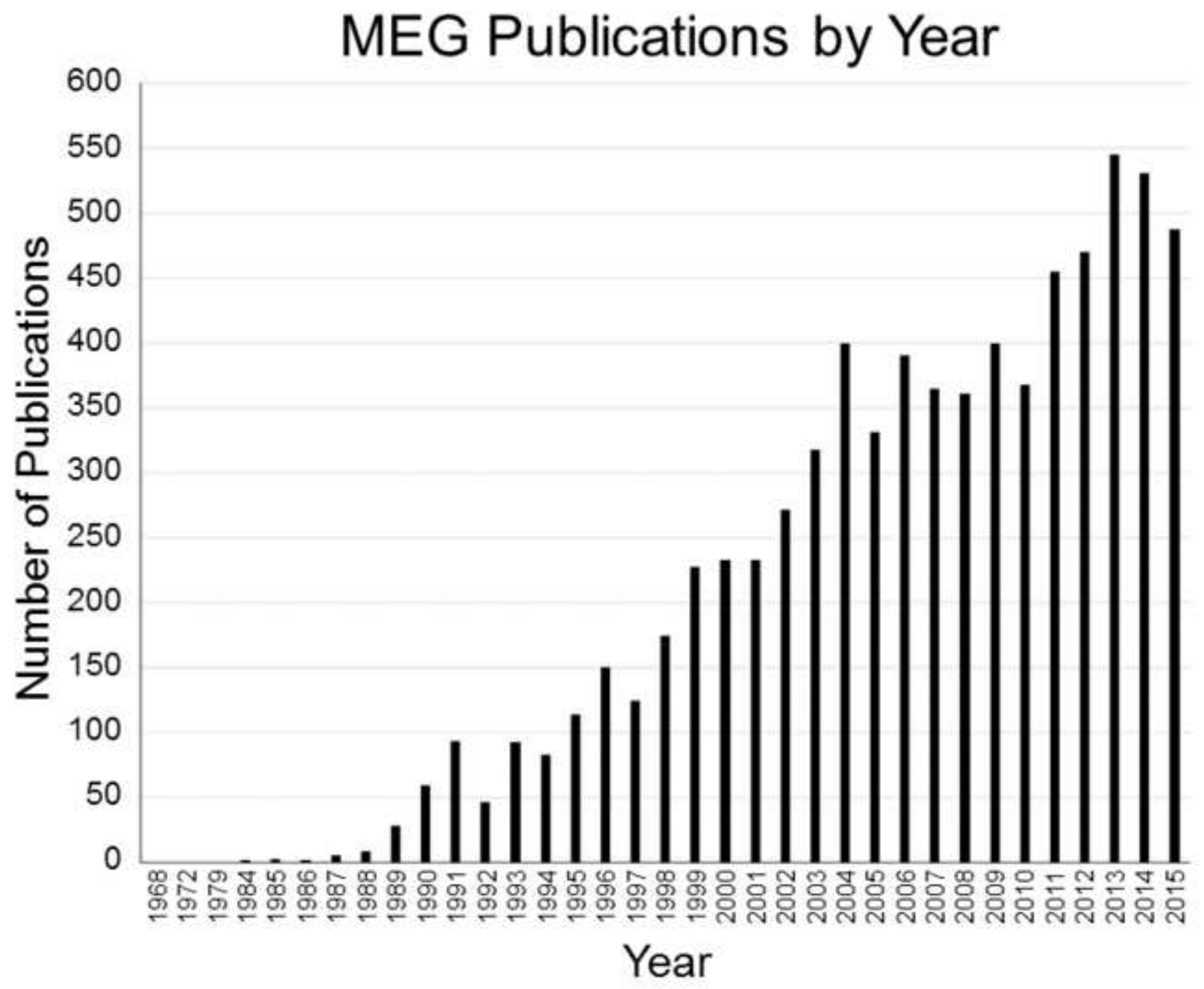



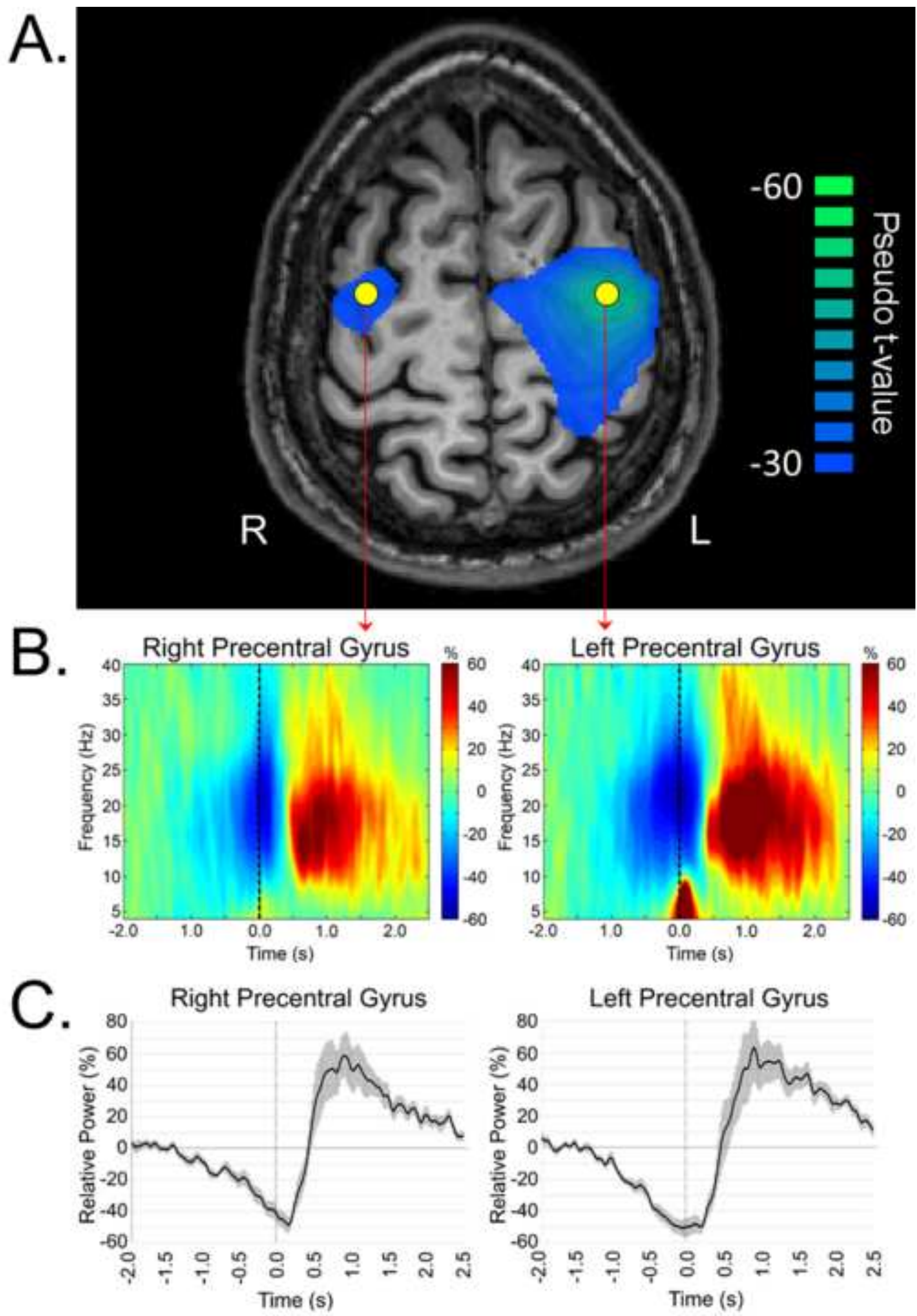


\section{DMN Pathway Strength by Group}

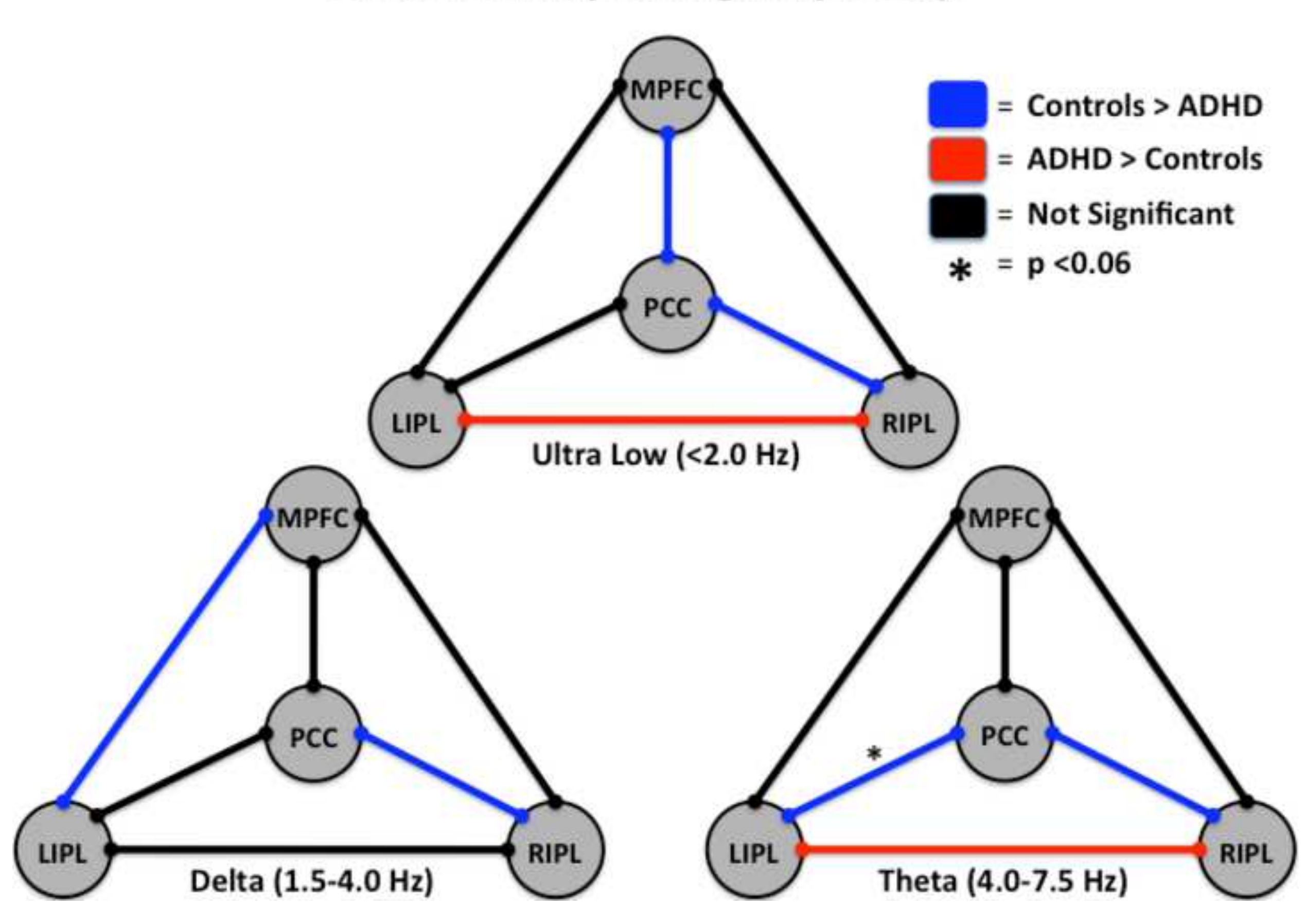




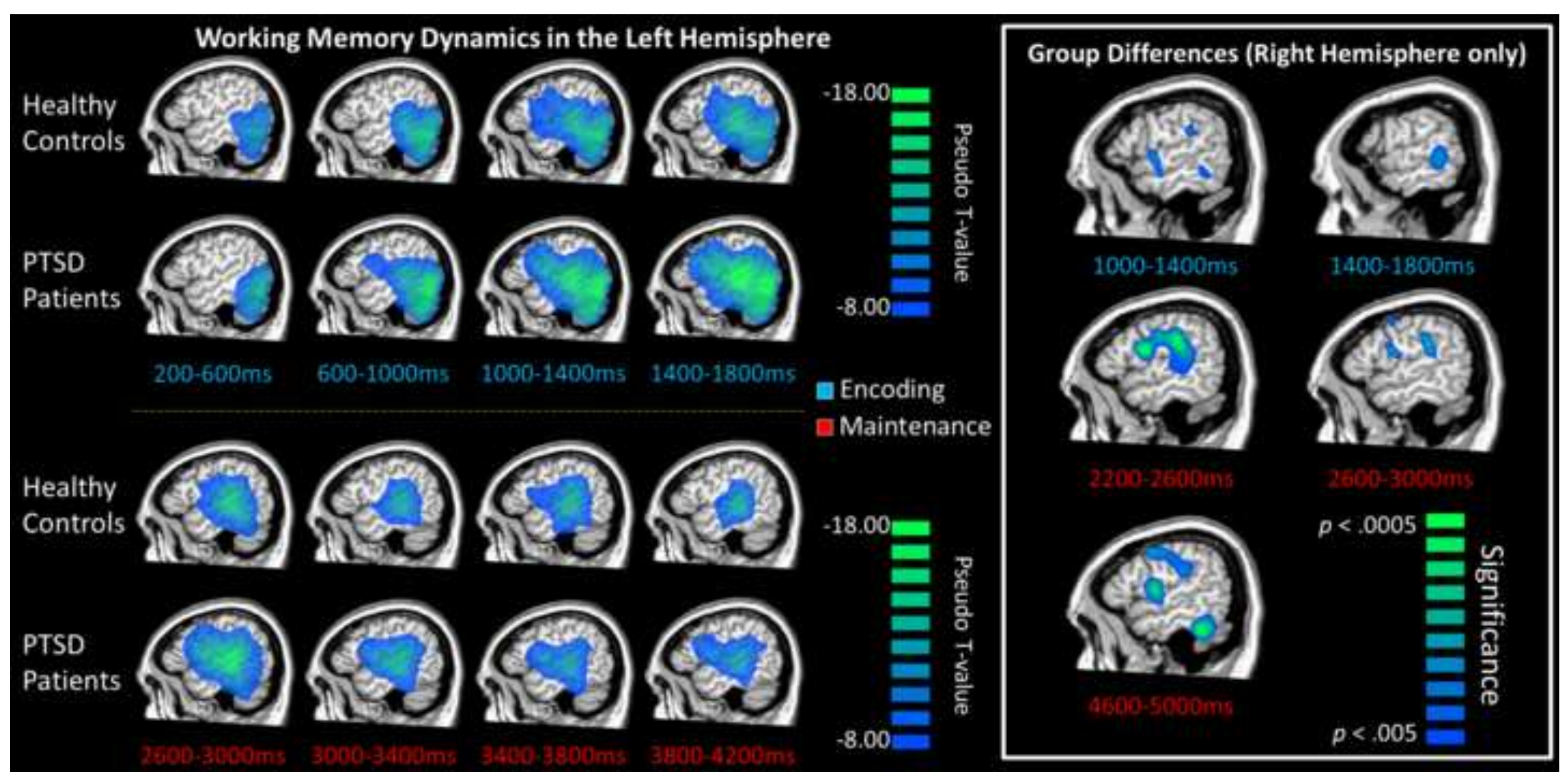



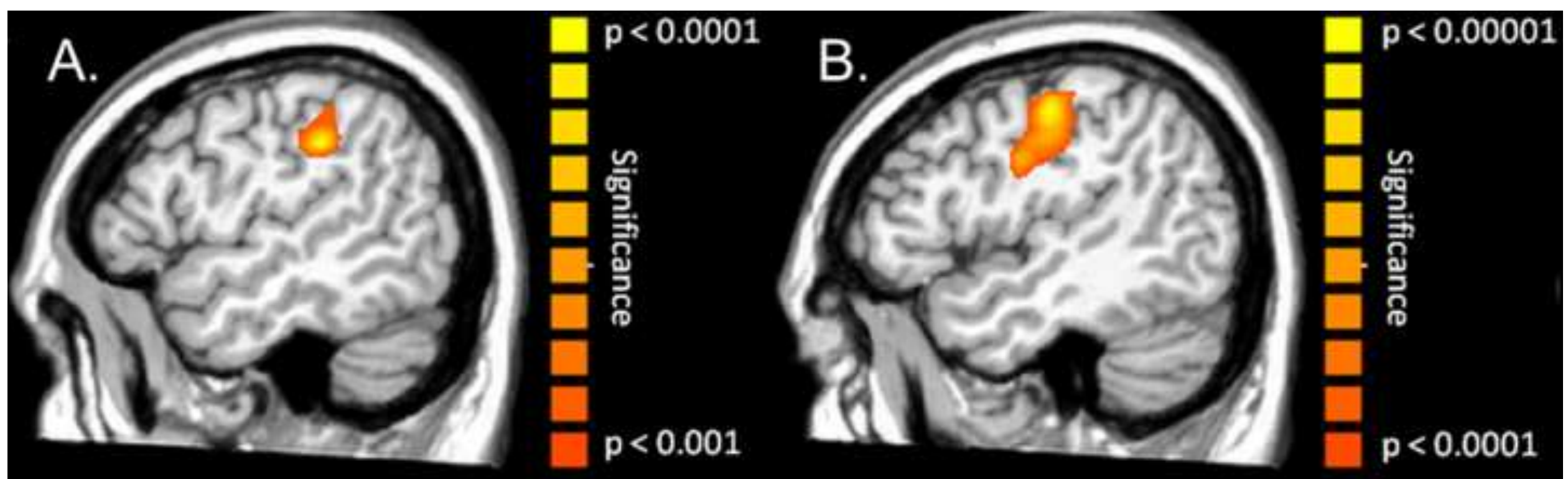

C.

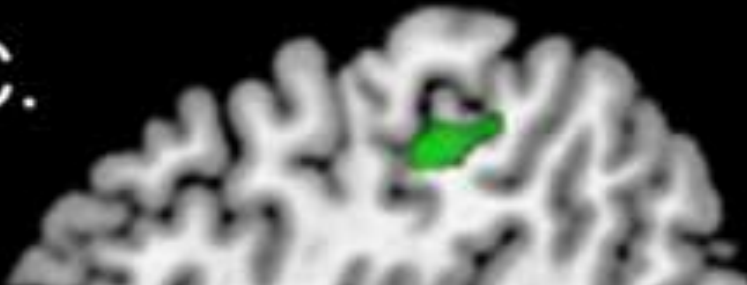
Esing
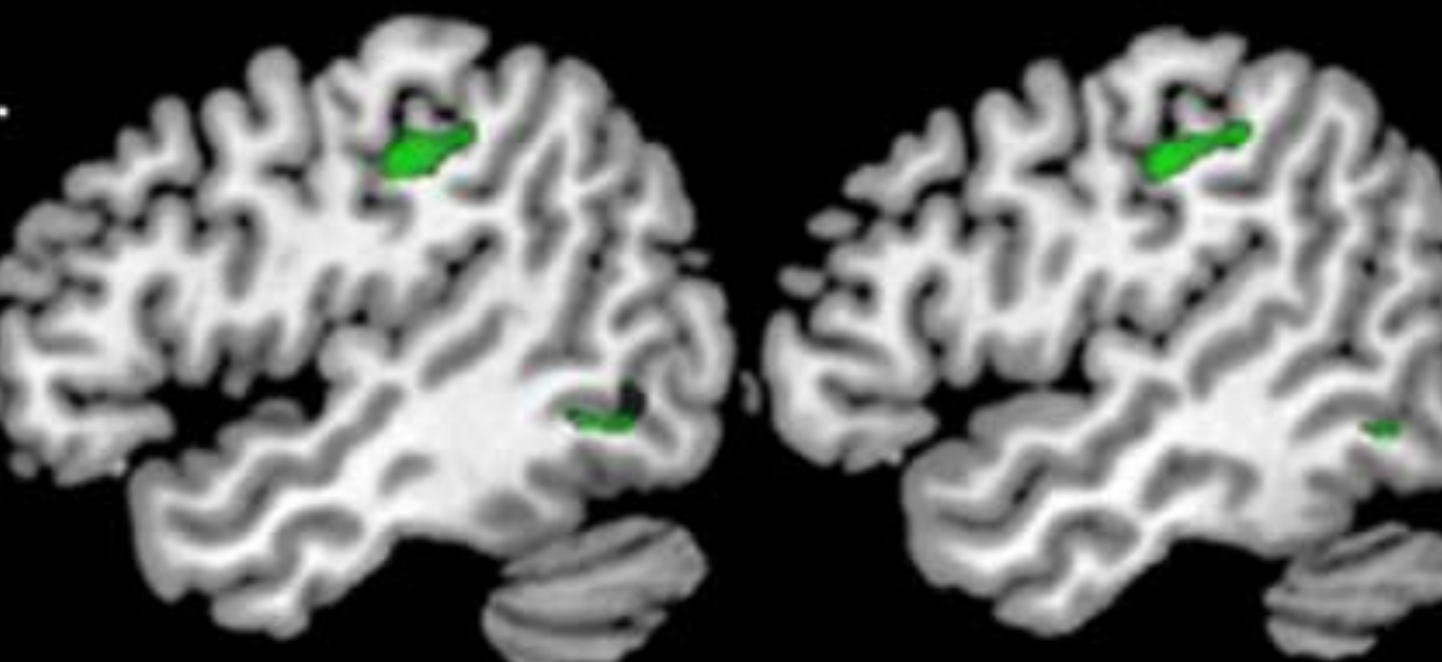
<ce 5 (n)
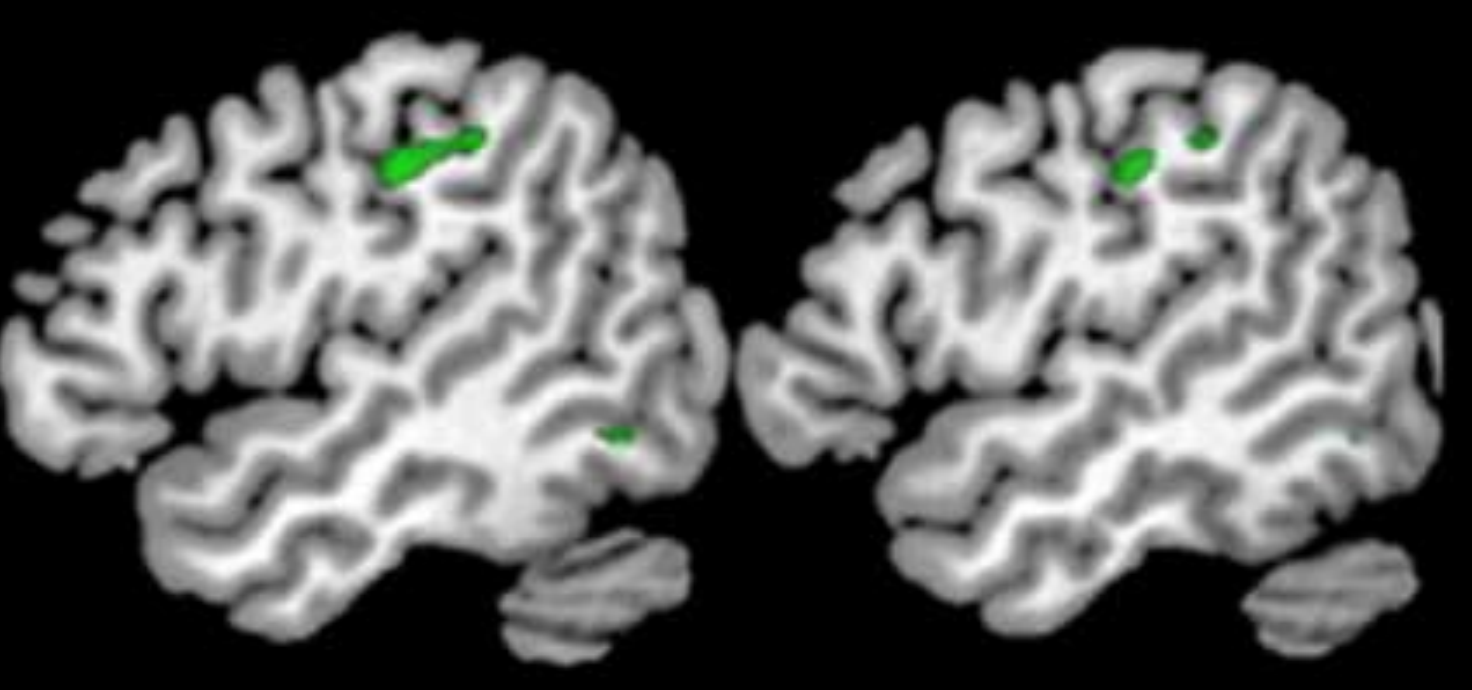

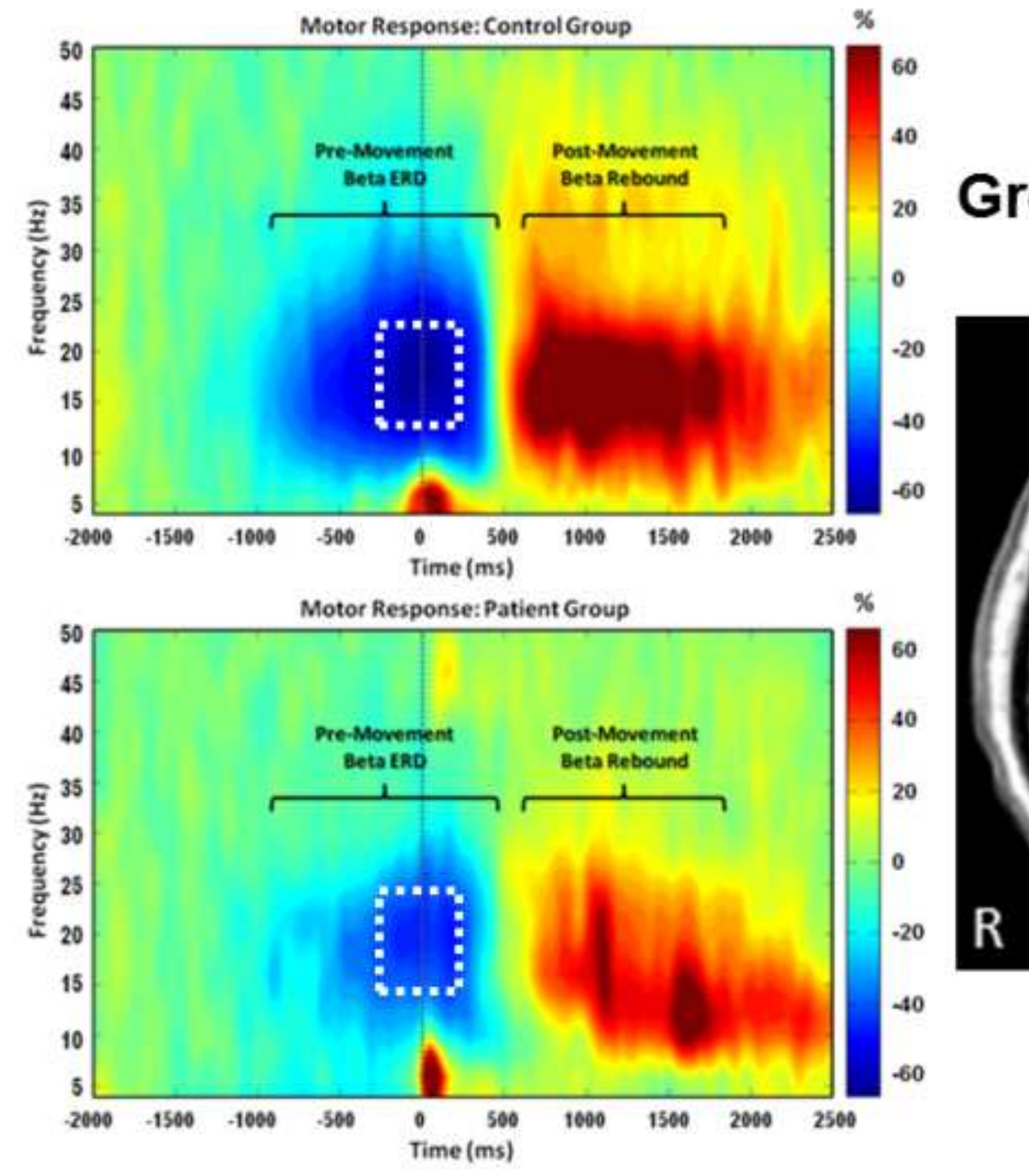

Group Difference: Beta ERD

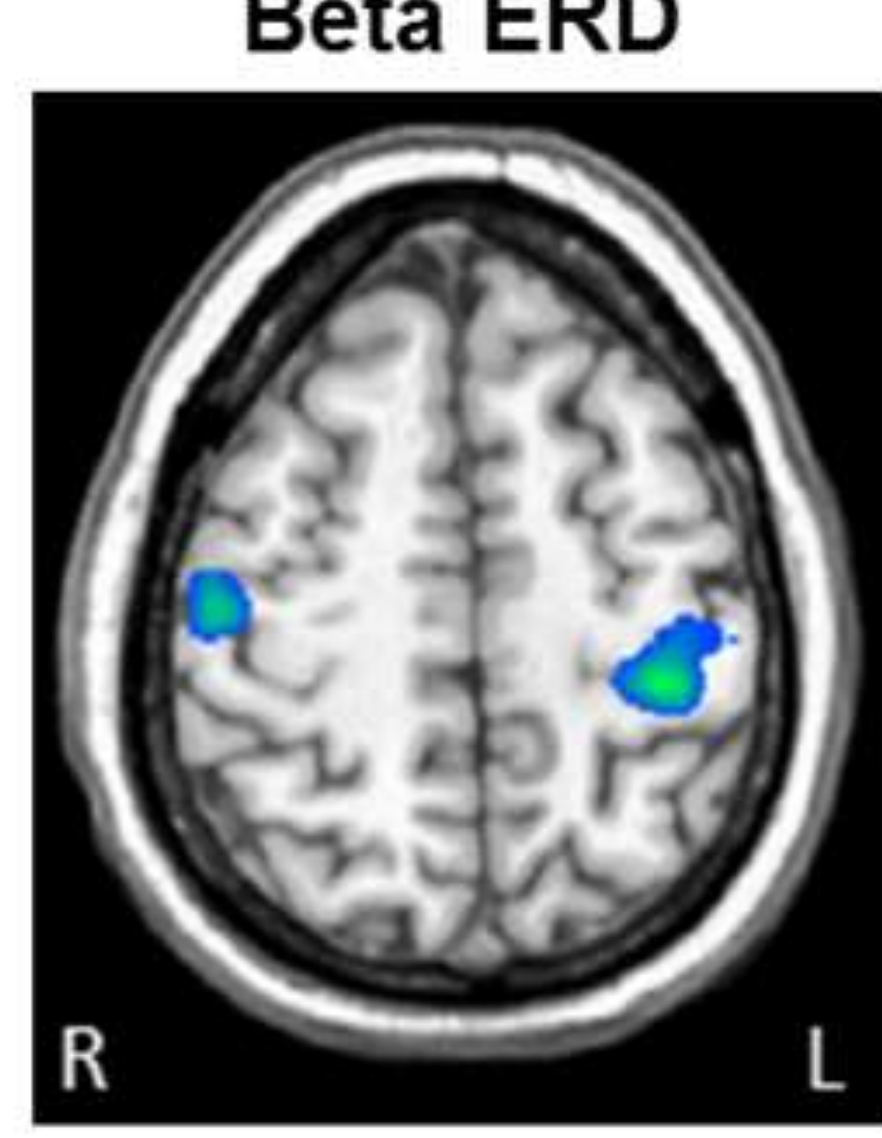


Age-Related Occipital Alpha Differences During Maintenance

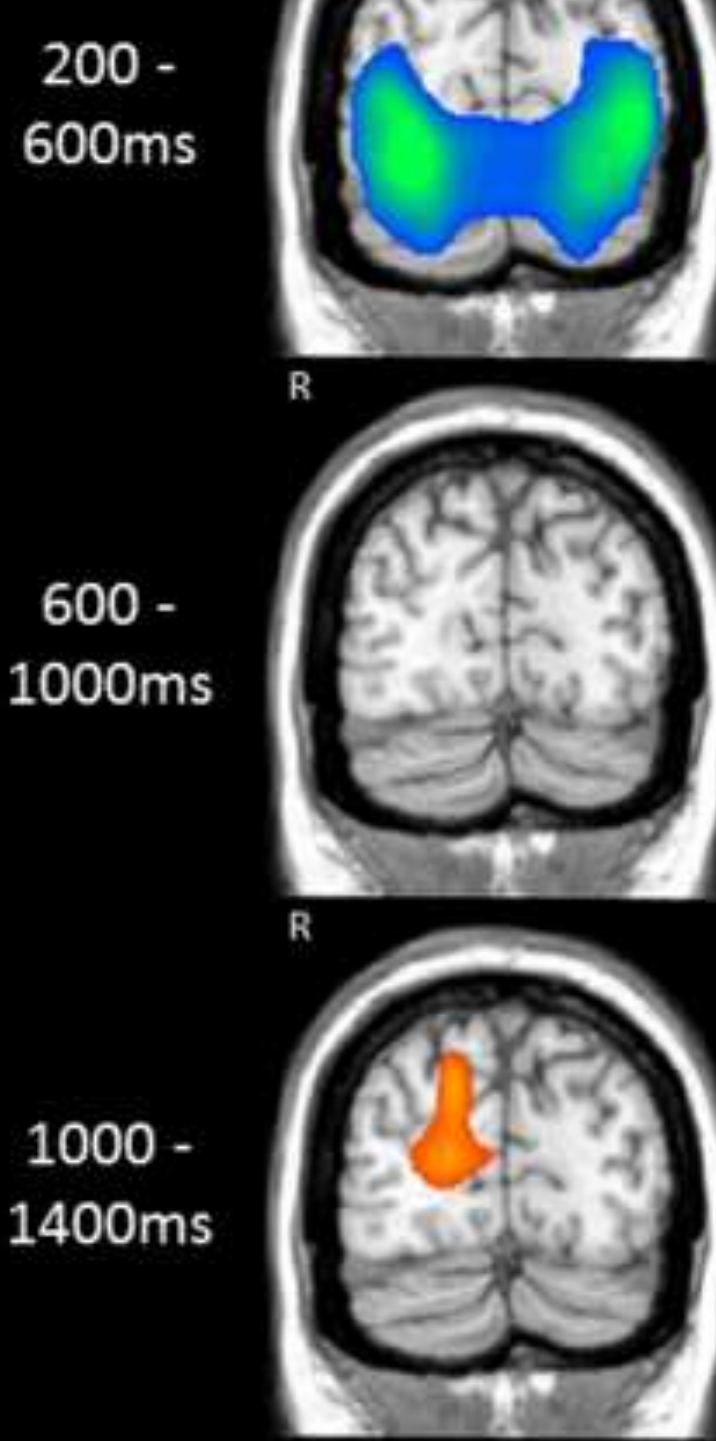

Younger Adults

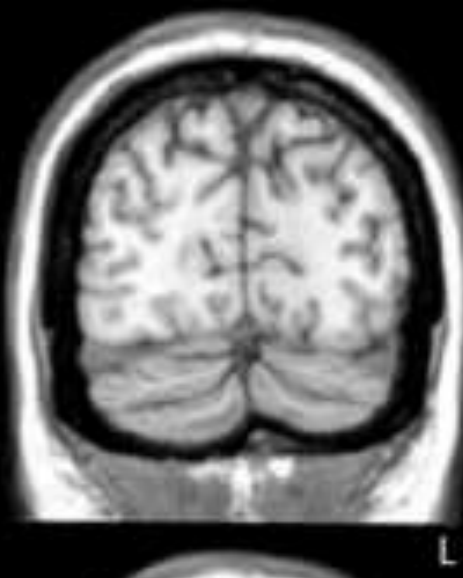

-15.80 드.
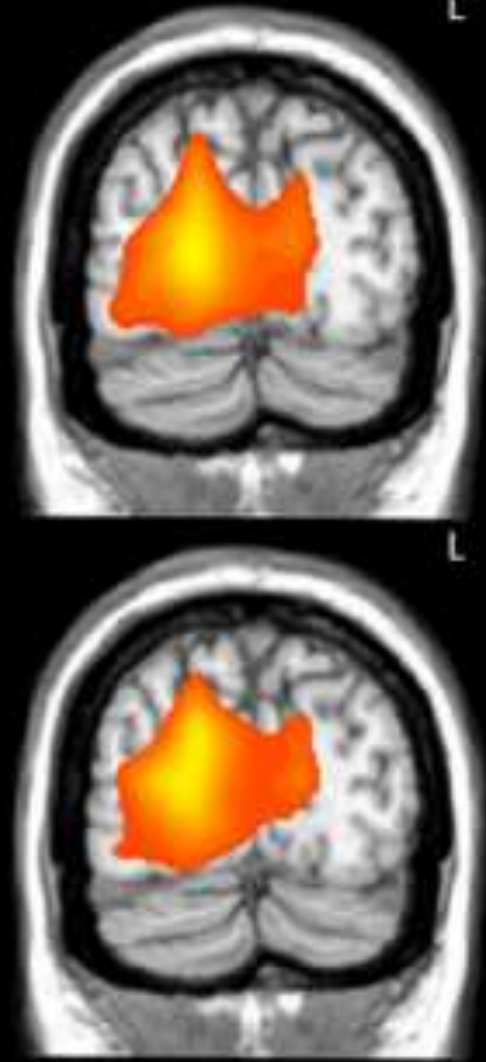

Older Adults
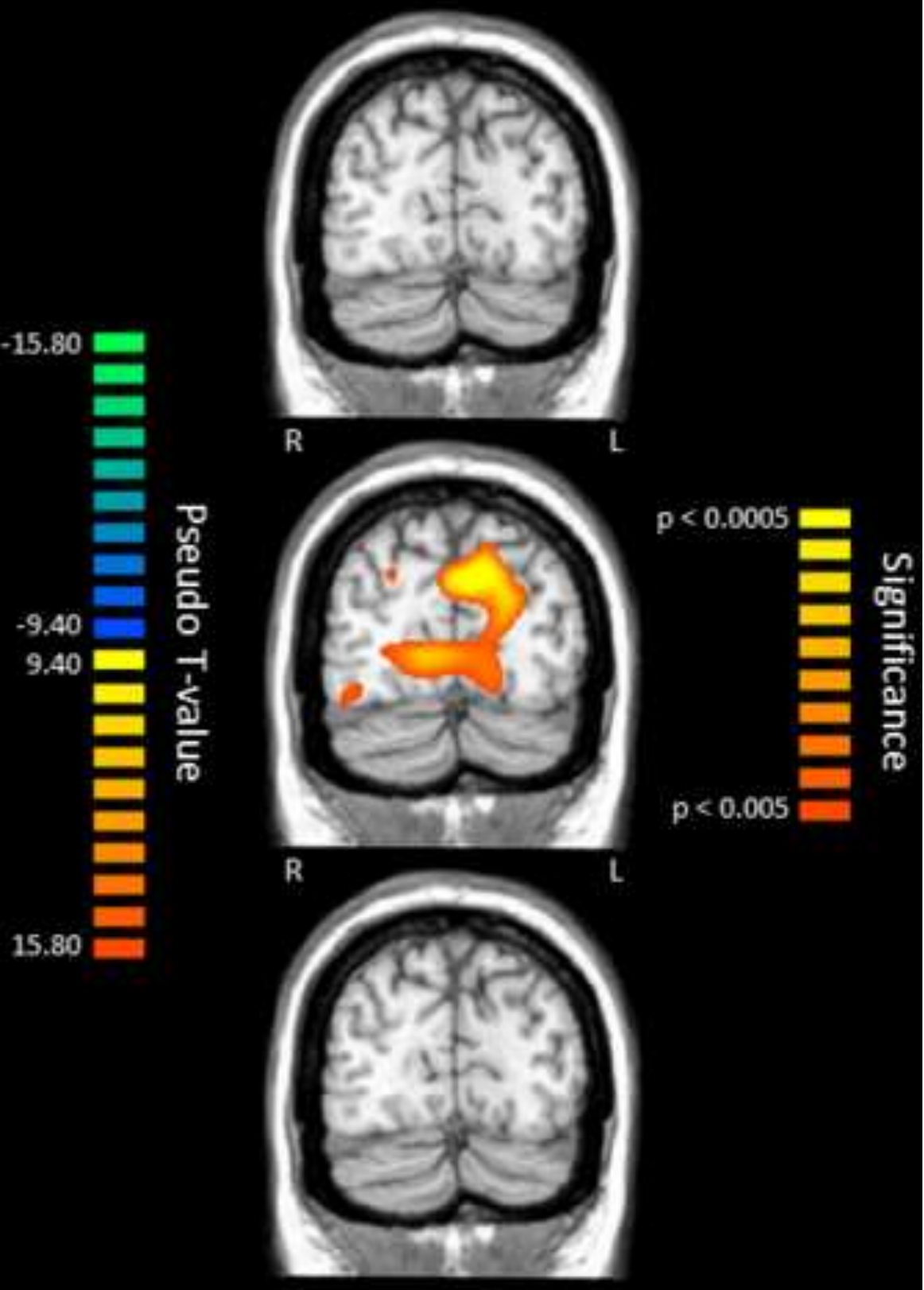

Group Differences 